\title{
Complex T-spherical fuzzy Dombi aggregation operators and their applications in multiple-criteria decision-making
}

\author{
Faruk Karaaslan ${ }^{1}$ (D) Mohammed Allaw Dawood Dawood ${ }^{1}$
}

Received: 24 January 2021 / Accepted: 13 June 2021 / Published online: 11 July 2021

(c) The Author(s) 2021

\begin{abstract}
Complex fuzzy (CF) sets (CFSs) have a significant role in modelling the problems involving two-dimensional information. Recently, the extensions of CFSs have gained the attention of researchers studying decision-making methods. The complex T-spherical fuzzy set (CTSFS) is an extension of the CFSs introduced in the last times. In this paper, we introduce the Dombi operations on CTSFSs. Based on Dombi operators, we define some aggregation operators, including complex T-spherical Dombi fuzzy weighted arithmetic averaging (CTSDFWAA) operator, complex T-spherical Dombi fuzzy weighted geometric averaging (CTSDFWGA) operator, complex T-spherical Dombi fuzzy ordered weighted arithmetic averaging (CTSDFOWAA) operator, complex T-spherical Dombi fuzzy ordered weighted geometric averaging (CTSDFOWGA) operator, and we obtain some of their properties. In addition, we develop a multi-criteria decision-making (MCDM) method under the CTSF environment and present an algorithm for the proposed method. To show the process of the proposed method, we present an example related to diagnosing the COVID-19. Besides this, we present a sensitivity analysis to reveal the advantages and restrictions of our method.
\end{abstract}

Keywords Complex fuzzy set · Spherical fuzzy set · Complex T-spherical fuzzy set · Dombi operators · Decision-making

\section{Introduction}

The fuzzy set (FS) theory was inaugurated by Zadeh [1] in 1965 to handle modelling of some problems containing uncertain data in real life. Since FS theory is a very useful tool for modelling uncertainty, it has many applications in the modelling and solving of the problems in many fields such as medical science, data mining and clustering. An FS is characterized by a membership function (MF) $\mu$ from a set of the objects or elements considered in the universe to the interval $[0,1]$. In an FS, if the membership degree (MD) of an element $x$ is $\mu(x)$, then its non-membership degree (NMD) is $1-\mu(x)$, that is, in the FS, hesitation degree of an element is " 0 ". This is one of the limited aspects of FS in modelling real-life problems. To overcome these limitations, the intuitionistic FS (IFS) was suggested by Atanassov [2] as

Faruk Karaaslan

karaaslan.faruk@gmail.com

Mohammed Allaw Dawood Dawood

mohammadalhadeathi@gmail.com

1 Department of Mathematics, Faculty of Sciences, Çankırı Karatekin University, 18100 Çankırı, Turkey a generalization of FSs. An IFS is identified by two functions from a universal set to the interval $[0,1]$ called membership function (MF) $(\mu)$ and non-membership function (NMF) $(v)$. The summation of images under these two functions of an element cannot exceed 1. Therefore, IFS is not an appropriate tool for modeling in the situation $\mu(x)+v(x)>1$. To cope with this restriction, Yager $[3,4]$ introduced the concept of Pythagorean FS (PyFS) as an extension of IFS under condition $\mu^{2}(x)+v^{2}(x) \leq 1$. However, in the situation $0.9^{2}+0.5^{2}=1.06>1$, a PyFS is not sufficient for modelling. To eliminate this type of limitation, Yager [5] put forward the concept of q-rung orthopair FS in which $\mu^{q}(x)+v^{q}(x) \leq 1$. The neutral situation is not taken into account in the set theories we have mentioned so far, but this situation is important for the representation of human thinking. For this, Cuong [6,7] defined the concept of the Picture FS (PFS). A PFS is a useful tool for expressing how much an object provides a feature or how much a person has shared an idea because a PFS does a modelling considering cases of yes, abstention, no, and rejection. A PFS is characterized by three values from interval $[0,1]$ for each element $\mathrm{x}$ belonging to set containing considered elements, called MD $(\mu(x))$, abstinence degree (AD) or neutral degree $(\gamma(x))$ and, NMD

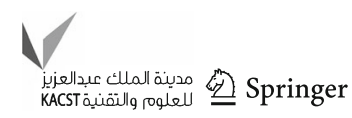


$(v(x))$ with the condition $0 \leq \mu(x)+\gamma(x)+v(x) \leq 1$. Despite the fact that PFS structure is a useful tool in many applications such as decision-making (DM) [8-14], similarity measure [15-19], correlation coefficient [20,21], and clustering [22,23], it is not sufficient in modelling of some problems because of constrain $0 \leq \mu(x)+\gamma(x)+v(x) \leq 1$. Therefore, the notion of spherical FS (SFS), which is an extension of PFS, was initiated by Gungogdu and Kahraman $[24,25]$ and the applications of the SFS to decision-making was studied on. An SFS has the constrain $0 \leq \mu^{2}(x)+$ $\gamma^{2}(x)+v^{2}(x) \leq 1$. Kahraman et al. [24] developed a DM method based on the TOPSIS method under the SF environment and presented an application of the developed method in the selection of hospital location. In an SFS, when MD, $\mathrm{NeD}$ and NMD of an element are taken as 0.6, 0.9 and 0.5, respectively, since $0.6^{2}+0.9^{2}+0.5^{2}=1.42>1$, condition $0 \leq \mu^{2}(x)+\gamma^{2}(x)+v^{2}(x) \leq 1$ is not satisfied. To model such situations, the T-spherical FS (T-SFS) was introduced by Mahmood et al. [26] as an extension of the SFS under condition $\mathrm{X}$ and some applications in medical diagnosis and DM problems under T-SF and SF environments were given by same researchers. After the works of Mahmood et al. [26], many researchers have studied applications of T-SFS and SFS. For example, Ullah et al. [27] proposed some novel similarity measures including cosine similarity measures, grey similarity measures, and set theoretic similarity measures for SFS and T-SFSs. Garg et al. [28] defined some new improved aggregation operators for T-SFSs and developed a DM approach to solve the multi-attribute DM (MADM) problems. Ullah et al. [29] introduced the some ordered weighted aggregation operators and hybrid aggregation operators of T-SFS and proposed an MADM method. Wu et al. [30] studied divergence measure of T-SFSs and gave the application in pattern recognition. Ullah et al. [31] defined the concept of interval-valued T-SFSs and their basic operations. They also described two aggregation operators for intervalvalued T-SF values and developed an MADM method for problem including evaluating companies to be made an investment. Liu et al. [32] proposed some novel operational laws for T-SPFNs and combine power average operator and with Murihead mean operator. They also developed some new aggregation operators. Guleria and Bajaj [33] defined some aggregation operations of T-Spherical fuzzy soft sets. Quek et al. [34] presented some new operational laws for T-spherical fuzzy sets and obtain some of their properties. Then, based on these new operations, they have proposed two types of Einstein aggregation operators called the Einstein interactive averaging aggregation operators and the Einstein interactive geometric aggregation operators. They also put forward a MADM method based on the defined aggregation operators. Munir et al. [35] studied on Einstein hybrid aggregation operators under T-SF environment and establish an MADM by integrating the proposed aggregation operators.
Ullah et al. [36] establish the correlation coefficient formula for T-SF values and presented an application in clustering. Also, T-spherical Fuzzy Hamacher Aggregation Operators were defined Ullah et al. [37]. Furthermore, they put forward an MADM method and gave the application of the method in a problem including evaluation of the performance of search and rescue robots. Garg et al. [38] introduced power aggregation operators for the T-spherical fuzzy sets (T-SFSs). Ju et al. [39] defined the T-SF interaction aggregation operators and based on these operators they developed TODIM method under T-SF environment. Chen et al. [40] studied on some generalized T-Spherical and group-Generalized fuzzy geometric aggregation operators with MADM method. Associated immediate probability (interactive) geometric aggregation operators of for T-spherical fuzzy sets were introduced by Munir et al. [41].

As mentioned above, FS models are important tools for modelling uncertain and incomplete data. But mentioned FS models do not suffice to express the periodic information or two-dimension phenomenon. To cope with this issue, the concept of complex FS (CFS) was put forward by Ramot et al. $[42,43]$. The basic idea in the definition given by Ramot is to extend the range of membership from $[0,1]$ to the unit circle in the complex plane. A CFS is characterized by membership function $\mu=r e^{i \omega}$ where $r$ is called amplitude term and it takes values from the interval $[0,1]$, and $\omega$ is called phase term (periodic term) and it lies in the interval $[0,2 \pi]$. The phase term has a very important role in defining the CF model. This is what makes the CF sets superior and distinct from other FS models. In a CFS, the membership value of an element is specified based on one amplitude term and one phase term. With this aspect, CFS is not enough to model the nonmembership degree. To avoid this restriction, Alkouri and Salleh [44] introduced complex intuitionistic FS (CIFS). A CIFS is identified by $\mathrm{MF}\left(\mu=r e^{i \omega}\right)$ and $\mathrm{NMF}\left(\nu=k e^{i \eta}\right)$ such that $0 \leq r+k \leq 1$ and $0 \leq \omega+\eta \leq 2 \pi$. Rani and Garg [45] developed a DM approach based on distance measure between CIFSs. Also, some researchers studied on aggregation operator of CIFS and DM methods [46-51]. Additionally, Ullah et al. [52] introduced the complex PyFS (CPFS) which is characterized by MF $\mu=r e^{i \omega}, \mathrm{NeF} \mu=s e^{i \theta}$, and NMF $v=k e^{i \eta}$ under the conditions $0 \leq r+s+k \leq 1$ and $0 \leq \frac{\omega}{2 \pi}+\frac{\theta}{2 \pi}+\frac{\eta}{2 \pi} \leq 1$ as a generalization of CIFSs. Liu et al. [53] defined the complex q-ROFS (CqROFS) and studied on aggregation operator of them.

Akram et al. [54] presented some aggregation operators under CPF environment based on Hamacher operations and developed an MCDM method. Liu et al. [55] introduced $\mathrm{CPF}$ power averaging and CPF power geometric operators under CPFSs environment and constructed an MCDM method based on the proposed operators. Additionally, the complex SFS (CSFS) was defined by Akram et al. [56] as a generalization of CPFS. They also introduced some aggre- 
gation operation based on Dombi t-norm and t-conorm. Ali et al. [57] defined the concept of complex T-spherical FS (CTSFS) and their aggregation operators. They also proposed an MADM method in CTSFSs. Akram et al. [58] defined some aggregation operators of CSFSs and developed an MCGDM method called CSF-VIKOR. Nasir et al. [59] introduced the notion of CTSF relations and presented some applications related to the economy and international trade.

Since aggregation operators (AOs) convert the whole data into a single value, AOs have a vital importance in DM problems. Dombi [60] designated Dombi operators with flexible operational variables. In solving the DM problems, many researchers used Dombi operations of IFS [61], Pythagorean fuzzy [62-64], PF [66], bipolar fuzzy [65], spherical fuzzy [67], complex Pythagorean [68], and CSF [69].

As seen above, the studies on the theoretical aspects of SFSs, T-SFSs, CSFSs and CTSFSs and their applications in decision-making based on aggregation operators have increased rapidly. The following points motivate us to present this paper:

- CFS and its generalizations have a very important role in decision-making problems containing two-dimensional information in real life. A TSFS comprehends a large amount of information as a generalization of the SFSs. However, it does not suffice in modelling an issue involving two-dimensional data. With this aspect, CTSFS has vital importance. A CTSFS is the generalization of theories like CFS, CIFS, CPFS, CPyFS and CSFS. Until now, there exists only one work [57] related to aggregation operators of CTSFS in the literature. Therefore, by considering the advantages of the Dombi operators, we develop some new aggregation operators based on Dombi t-norm and t-conorm to use in modelling a problem involving two-dimensional data.

- Set-theoretical operators are an important tool for modelling some problems, in the literature, there is not any study related to set-theoretical operations of CTSFS. To fill this gap in the literature, we define the set-theoretical operations of CTSFSs.

- In literature, there is only one study related to score and accuracy functions of CTSFNs and these functions have some drawbacks, we pointed out these drawbacks and define novel score and accuracy functions free from specified drawbacks.

- We see that works related to aggregation operators of SFS, TSFS and CTSFSs are based on the hypothetical data in general. In this study, one of our aims is to develop a decision-making method by considering the advantages of the Dombi operators and presenting an application including real data that aims to diagnose COVID-19 patients.
This article is organized as follows: the next section recalls the required definitions in the following sections as SFS, TSFS, CTSFSs and Dombi operations. Also, new score and accuracy functions and set-theoretical operation are defined. The subsequent section defines Dombi operations of complex T-spherical fuzzy numbers and provides their examples, and related operations of these operators are obtained for introduced aggregation operators. Then the MCDM method and its application are presented. Before the final section, sensitivity analyses and discussion related to obtained results from the application of the proposed method are given. The final section mentions the conclusions and planned studies.

\section{Preliminaries}

This section reminds the definitions of CFS, CIFS, CPyFS, CPFS, SFS, T-SFS and CTSFSs.

Definition 1 [42] Let $\mathfrak{X}$ be a nonempty set. A complex fuzzy set (CFS) $\Im$ is defined as

$\mathcal{F}=\left\{\left(\mathfrak{x}, \tilde{\alpha}_{\mathcal{F}}(\mathfrak{x})\right): \mathfrak{x} \in \mathfrak{X}\right\}$,

where $\tilde{\alpha}_{\mathcal{F}}(\mathfrak{x})$ is called membership functions of CFS $\mathcal{F}$ and receive all lying within the unit circle in the complex plane. Thus, it can be expressed as $\tilde{\alpha}_{\mathcal{F}}(\mathfrak{x})=\alpha_{\mathcal{F}}(\mathfrak{x}) e^{i 2 \pi \omega_{\alpha \mathcal{F}}(\mathfrak{x})}$, and it denotes a complex-valued grade of membership of $\mathfrak{x} \in \mathfrak{X}$ to (CFS) $\mathfrak{~}$. Here $i=\sqrt{-1}$ and for all $\mathfrak{x} \in \mathfrak{X}, 0 \leq \alpha_{\mathcal{F}}(\mathfrak{x}) \leq 1$, and $0 \leq \varpi_{\alpha_{\mathcal{F}}}(\mathfrak{x}) \leq 1$.

Definition 2 [44] Let $\mathfrak{X}$ be a nonempty set. A complex intuitionistic fuzzy set (CIFS) $\mathcal{I}$ is defined as:

$\mathcal{I}=\left\{\left(\mathfrak{x}, \tilde{\alpha}_{\mathcal{I}}(\mathfrak{x}), \tilde{\gamma}_{\mathcal{I}}(\mathfrak{x})\right): \mathfrak{x} \in \mathfrak{X}\right\}$,

where $\tilde{\alpha}_{\mathcal{I}}(\mathfrak{x})$ and $\tilde{\gamma}_{\mathcal{I}}(\mathfrak{x})$ are called membership function and non-membership function of CIFS $\mathcal{I}$, respectively. They receive all lying within the unit circle in the complex plane.

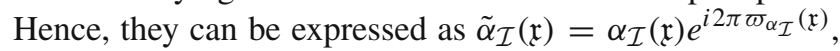
and $\tilde{\gamma}_{\mathcal{I}}(\mathfrak{x})=\gamma_{\mathcal{I}}(\mathfrak{x}) e^{i 2 \pi \varpi_{\gamma_{\mathcal{I}}}(\mathfrak{x})}$, where they denote the complex-valued grades of membership and non-membership of $\mathfrak{x} \in \mathfrak{X}$ to CIFS $\mathcal{I}$, respectively. Here $i=\sqrt{-1}$, for all $\mathfrak{x} \in$ $\mathfrak{X}, 0 \leq \alpha_{\mathcal{I}}(\mathfrak{x})+\gamma_{\mathcal{I}}(\mathfrak{x}) \leq 1$, and $0 \leq \varpi_{\alpha_{\mathcal{I}}}(\mathfrak{x})+\varpi_{\gamma_{\mathcal{I}}}(\mathfrak{x}) \leq 1$.

Definition 3 [52] Let $\mathfrak{X}$ be a nonempty set. A complex pythagorean fuzzy set (CPyFS) $\mathfrak{P}$ is defined as

$\mathfrak{P}=\left\{\left(\mathfrak{x}, \tilde{\alpha}_{\mathfrak{P}}, \tilde{\gamma}_{\mathfrak{P}}(\mathfrak{x})\right): \mathfrak{x} \in \mathfrak{X}\right\}$,

where $\tilde{\alpha}_{\mathfrak{P}}(\mathfrak{x})$ and $\tilde{\gamma}_{\mathfrak{P}}(\mathfrak{x})$ are called complex-valued membership function and non-membership function of CPyFS $\mathfrak{P}$, respectively. They receive all lying within the unit circle in the complex plane. Thus, they can be expressed as 
$\tilde{\alpha}_{\mathfrak{P}}(\mathfrak{x})=\alpha_{\mathfrak{P}}(\mathfrak{x}) e^{i 2 \pi \omega_{\alpha_{\mathfrak{P}}}(\mathfrak{x})}$, and $\tilde{\gamma}_{\mathfrak{P}}(\mathfrak{x})=\gamma_{\mathfrak{P}}(\mathfrak{x}) e^{i 2 \pi \varpi_{\gamma_{\mathfrak{P}}}(\mathfrak{x})}$, where they denote the complex-valued grades of membership and non-membership of $\mathfrak{x} \in \mathfrak{X}$ to CPyFS $\mathfrak{P}$, respectively. Here $i=\sqrt{-1}$ and for all $\mathfrak{x} \in \mathfrak{X}, 0 \leq \alpha_{\mathcal{I}}^{2}(\mathfrak{x})+\gamma_{\mathcal{I}}^{2}(\mathfrak{x}) \leq 1$, and $0 \leq \varpi_{\alpha_{\mathfrak{P}}}^{2}(\mathfrak{x})+\varpi_{\gamma_{\mathfrak{P}}}^{2}(\mathfrak{x}) \leq 1$.

Definition 4 [54] Let $\mathfrak{X}$ be a nonempty set. A complex picture fuzzy set (CPFS) $\mathcal{P}$ is defined as

$\mathcal{P}=\left\{\left(\mathfrak{x}, \tilde{\alpha}_{\mathcal{P}}(\mathfrak{x}), \tilde{\beta}_{\mathcal{P}}(\mathfrak{x}), \tilde{\gamma}_{\mathcal{P}}(\mathfrak{x})\right): \mathfrak{x} \in \mathfrak{X}\right\}$,

where $\alpha_{\mathcal{P}}(\mathfrak{x}), \beta_{\mathcal{P}}(\mathfrak{x})$, and $\gamma_{\mathcal{P}}(\mathfrak{x})$ are called membership, neutral membership, and non-membership function of the CPFS $\mathcal{P}$, respectively. They receive all lying within the unit circle in the complex plane. Thus, they can be expressed as $\tilde{\alpha}_{\mathcal{P}}(\mathfrak{x})=\alpha_{\mathcal{P}}(\mathfrak{x}) e^{i 2 \pi \varpi_{\alpha_{\mathcal{P}}}(\mathfrak{x})}, \tilde{\beta}_{\mathcal{P}}(\mathfrak{x})=\beta_{\mathcal{P}}(\mathfrak{x}) e^{i 2 \pi \varpi_{\beta_{\mathcal{P}}}(\mathfrak{x})}$, and $\tilde{\gamma}_{\mathcal{P}}(\mathfrak{x})=\gamma_{\mathcal{P}}(\mathfrak{x}) e^{i 2 \pi \varpi_{\gamma_{\mathcal{P}}}(\mathfrak{x})}$, where they denote the complexvalued grades of membership and non-membership of $\mathfrak{x} \in \mathfrak{X}$ to CIFS $\mathcal{P}$, respectively. Here $i=\sqrt{-1}$, and for all $\mathfrak{x} \in \mathfrak{X}$, $0 \leq \alpha_{\mathcal{P}}(\mathfrak{x})+\beta_{\mathcal{P}}(\mathfrak{x})+\gamma_{\mathcal{P}}(\mathfrak{x}) \leq 1$, and $0 \leq \varpi_{\alpha_{\mathcal{P}}}(\mathfrak{x})+\varpi_{\beta_{\mathcal{P}}}(\mathfrak{x})+$ $\varpi_{\gamma_{\mathcal{P}}}(\mathfrak{x}) \leq 1$.

Definition $5[24,25]$ Let $\mathfrak{X}$ be a non-empty set. A spherical fuzzy set (SFS) $\mathcal{A}$ is defined over $\mathfrak{X}$ as follows:

$$
\begin{aligned}
\mathcal{A}= & \left\{\left(\mathfrak{x}, \alpha_{\mathcal{A}}(\mathfrak{x}),\right.\right. \\
& \left.\beta_{\mathcal{A}}(\mathfrak{x}), \gamma_{\mathcal{A}}(\mathfrak{x})\right): 0 \\
\leq & \alpha_{\mathcal{A}}^{2}(\mathfrak{x})+\beta_{\mathcal{A}}^{2}(\mathfrak{x}) \\
& \left.+\gamma_{\mathcal{A}}^{2}(\mathfrak{x}) \leq 1, \mathfrak{x} \in \mathfrak{X}\right\} .
\end{aligned}
$$

Here the function $\alpha_{\mathcal{A}}: \mathfrak{X} \rightarrow[0,1]$ expresses MF, $\beta_{\mathcal{A}}: \mathfrak{X} \rightarrow$ $[0,1]$ expresses NeMF, and $\gamma_{\mathcal{A}}: X \rightarrow[0,1]$ expresses NMF of the SFS $\mathcal{A}$.

The concept of T-spherical fuzzy set was introduced by Mahmood et al. [26] as a generalization of the SFSs, as follows:

Definition 6 [26] Let $\mathfrak{X}$ be a nonempty set. A T-spherical fuzzy (TSF) set (TSFS) is defined over $\mathfrak{X}$ as follows:

$$
\begin{aligned}
\mathcal{T}= & \left\{\left(\mathfrak{x}, \alpha_{\mathcal{T}}(\mathfrak{x}), \beta_{\mathcal{T}}(\mathfrak{x}),\right.\right. \\
& \left.\gamma_{\mathcal{T}}(\mathfrak{x})\right): 0 \leq \\
& \alpha_{\mathcal{T}}^{q}(\mathfrak{x})+\beta_{\mathcal{T}}^{q}(\mathfrak{x}) \\
& \left.+\gamma_{\mathcal{T}}^{q}(\mathfrak{x}) \leq 1, \mathfrak{x} \in \mathfrak{X}\right\} .
\end{aligned}
$$

Here the function $\alpha_{\mathcal{A}}: \mathfrak{X} \rightarrow[0,1], \beta_{\mathcal{T}}: \mathfrak{X} \rightarrow[0,1]$, and $\gamma_{\mathcal{A}}: \mathfrak{X} \rightarrow[0,1]$ express MF, NeMF, and NMF of the TSFS $\mathcal{T}$, respectively.
Definition 7 [57] Let $\mathfrak{X}$ be an initial universe different from empty set. A complex T-spherical fuzzy (CTSF) set (CTSFS) is defined as follows:

$\digamma=\left\{\left(\mathfrak{x}, \alpha_{\digamma}(\mathfrak{x}), \beta_{\digamma}(\mathfrak{x}), \gamma_{\digamma}(\mathfrak{x})\right): \mathfrak{x} \in \mathfrak{X}\right\}$

Here $\alpha_{\digamma}(\mathfrak{x})=\alpha_{\digamma}(\mathfrak{x}) e^{i 2 \pi \varpi_{\beta_{\digamma}(\mathfrak{x})}}, \beta_{\digamma}(\mathfrak{x})=\beta_{\digamma}(\mathfrak{x}) e^{i 2 \pi \varpi_{\beta_{\digamma}(\mathfrak{x})}}$, and $\gamma_{\digamma}(\mathfrak{x})=\gamma_{\digamma}(\mathfrak{x}) e^{i 2 \pi \omega_{\gamma_{\digamma}(\mathfrak{x})}}$ denote the membership grades of truth, abstinence, and falsity such that $0 \leq \alpha_{\digamma}^{q}(\mathfrak{x})+$ $\beta_{\digamma}^{q}(\mathfrak{x})+\gamma_{\digamma}^{q}(\mathfrak{x}) \leq 1$ and $0 \leq \varpi_{\alpha_{\digamma}(\mathfrak{x})}^{q}+\varpi_{\beta_{\digamma}(\mathfrak{x})}^{q}+\varpi_{\gamma_{\digamma}(\mathfrak{x})}^{q} \leq 1$. Furthermore,

$\mathfrak{H}_{\digamma}(\mathfrak{x})=\sqrt[q]{1-\alpha_{\digamma}(\mathfrak{x})^{q}-\beta_{\digamma}(\mathfrak{x})^{q}-\alpha_{\digamma}(\mathfrak{x})^{q}}$
$e^{\sqrt[q]{\left(1-\varpi_{\alpha_{\digamma}(\mathfrak{x})}^{q}-\varpi_{\beta_{\digamma}(\mathfrak{x})}^{q}-\varpi_{\gamma_{\digamma}(\mathfrak{x})}^{q}\right.}}$ expresses the complex hesitancy grade of $\mathfrak{x}$.

For convenience, $\digamma=\left(\alpha_{k} e^{i 2 \pi \omega_{\alpha_{k}}}, \beta_{k} e^{i 2 \pi \omega_{\beta_{k}}}, \gamma_{k} e^{i 2 \pi \varpi_{\gamma_{k}}}\right)$ is called complex T-spherical fuzzy number (CTSFN).

Ali et al. [57] defined the score functions for the CTSFNs by taking absolute value of formula given the following definitions.

$$
\begin{aligned}
& \Omega(\digamma)=\frac{1}{2}\left|\left(\alpha^{q}-\beta^{q}-\gamma^{q}\right)+\left(\varpi_{\alpha^{q}}-\varpi_{\beta^{q}}-\varpi_{\gamma^{q}}\right)\right| \\
& \mho(\digamma)=\frac{1}{2}\left|\left(\alpha^{q}+\beta^{q}+\gamma^{q}\right)+\left(\varpi_{\alpha^{q}}+\varpi_{\beta^{q}}+\varpi_{\gamma^{q}}\right)\right| .
\end{aligned}
$$

When we consider the CTSFNs $\left(1 e^{i 2 \pi 1}, 0 e^{i 2 \pi 0}, 0 e^{i 2 \pi 0}\right)$ and $\left(0 e^{i 2 \pi 1}, 0 e^{i 2 \pi 0}, 1 e^{i 2 \pi 1}\right)$, their score values are 1 . So, we need to use the accuracy function, but their accuracy values are 1. This is a weak aspect of the proposed score and accuracy functions. Therefore, we define the following score and accuracy functions.

Definition 8 Let $\digamma=\left(\alpha e^{i 2 \pi \varpi_{\alpha}}, \beta e^{i 2 \pi \varpi_{\beta}}, \gamma e^{i 2 \pi \omega_{\gamma}}\right)$ is a CTSFN. The score function $\Omega(\digamma)$ and accuracy function $\mho(\digamma)$ of $\digamma$ are formulated as follows:

$\Omega(\digamma)=\frac{1}{4}\left(2+\left(\alpha^{q}-\beta^{q}-\gamma^{q}\right)+\left(\varpi_{\alpha^{q}}-\varpi_{\beta^{q}}-\varpi_{\gamma^{q}}\right)\right)$

$\mho(\digamma)=\frac{1}{4}\left(2+\left(\alpha^{q}+\beta^{q}+\gamma^{q}\right)+\left(\varpi_{\alpha^{q}}+\varpi_{\beta^{q}}+\varpi_{\gamma^{q}}\right)\right)$.

Definition 9 Let $\digamma_{1}=\left(\alpha_{1} e^{i 2 \pi \omega_{\alpha_{1}}}, \beta_{1} e^{i 2 \pi \varpi_{\beta_{1}}}, \gamma_{1} e^{i 2 \pi \sigma_{\gamma_{1}}}\right)$ and $\digamma_{2}=\left(\alpha_{2} e^{i 2 \pi \omega_{\alpha_{2}}}, \beta_{2} e^{i 2 \pi \omega_{\beta_{2}}}, \gamma_{2} e^{i 2 \pi \omega_{\gamma_{2}}}\right)$ are two CTSFNs. For the comparison of $\digamma_{1}$ and $\digamma_{2}$,

$-\digamma_{1} \succeq \digamma_{2}\left(\digamma_{1}\right.$ is superior to $\left.\digamma_{2}\right)$ if $\Omega\left(\digamma_{1}\right)>\Omega\left(\digamma_{2}\right)$;

- if $\Omega\left(\digamma_{1}\right)=\Omega\left(\digamma_{2}\right)$, then

$-\digamma_{1} \succeq \digamma_{2}\left(\digamma_{1}\right.$ is superior to $\left.\digamma_{2}\right)$ if $\mho\left(\digamma_{1}\right)>\mho\left(\digamma_{2}\right)$;

$-\digamma_{1} \sim \digamma_{2}\left(\digamma_{1}\right.$ is equivalent to $\left.\digamma_{2}\right)$ if $\mho\left(\digamma_{1}\right)=$ $\mho\left(\digamma_{2}\right)$. 
Definition 10 Let $\digamma_{1}=\left\{\left(\mathfrak{x}, \alpha_{1}(\mathfrak{x}) e^{i 2 \pi \varpi_{\alpha_{1}}(\mathfrak{x})}, \beta_{1}(\mathfrak{x})\right.\right.$ $\left.\left.e^{i 2 \pi \omega_{\beta_{1}}(\mathfrak{x})}, \gamma_{1}(\mathfrak{x}) e^{i 2 \pi \varpi_{\gamma_{1}}(\mathfrak{x})}\right) \quad: \quad \mathfrak{x} \quad \in \quad \mathfrak{X}\right\} \quad$ and $\digamma_{2}=\left\{\left(\mathfrak{x}, \alpha_{2}(\mathfrak{x}) e^{i 2 \pi \omega_{\alpha_{2}(\mathfrak{x})}}, \beta_{2}(\mathfrak{x}) e^{i 2 \pi \omega_{\beta_{2}(\mathfrak{x})}}, \gamma_{2}(\mathfrak{x}) e^{i 2 \pi \omega_{\gamma_{2}(\mathfrak{x})}}\right):\right.$ $\mathfrak{x} \in \mathfrak{X}\}$ be any two CTSFSs. Then

1. $\digamma_{1} \preceq \digamma_{2}$ iff $\alpha_{1}(\mathfrak{x}) \leq \alpha_{2}(\mathfrak{x}), \quad \beta_{1}(\mathfrak{x}) \leq \beta_{2}(\mathfrak{x}), \gamma_{1}(\mathfrak{x}) \geq$ $\gamma_{2}(\mathfrak{x})$ and $\varpi_{\alpha_{1}(\mathfrak{x})} \leq \varpi_{\alpha_{2}(\mathfrak{x})}, \varpi_{\beta_{1}(\mathfrak{x})} \leq \varpi_{\beta_{2}(\mathfrak{x})}, \varpi_{\gamma_{1}(\mathfrak{x})} \geq$ $\varpi_{\gamma_{2}(\mathfrak{x})}$, for all $\mathfrak{x} \in \mathfrak{X}$.

2. $\left(\digamma_{1}\right)^{c}=\left\{\left(\mathfrak{x}, \gamma_{1}(\mathfrak{x}) e^{i 2 \pi \sigma_{\gamma_{1}(\mathfrak{x})}}, \beta_{1}(\mathfrak{x}) e^{i 2 \pi \varpi_{\beta_{1}(\mathfrak{x})}}, \alpha_{1}(\mathfrak{x})\right.\right.$ $\left.e^{\left.i 2 \pi \varpi_{\alpha_{1}(\mathfrak{x})}\right)}: \mathfrak{x} \in \mathfrak{X}\right\}$.

3.

$$
\begin{aligned}
& \digamma_{1} \cup \digamma_{2}=\left\{\left(\mathfrak{x}, \max \left\{\alpha_{1}(\mathfrak{x}), \alpha_{2}(\mathfrak{x})\right\} e^{i 2 \pi \max \left\{\varpi_{\alpha_{1}(\mathfrak{x})}, \varpi_{\alpha_{2}(\mathfrak{x})}\right\}},\right.\right. \\
& \min \left\{\beta_{1}(\mathfrak{x}), \beta_{2}(\mathfrak{x})\right\} e^{i 2 \pi \min \left\{\varpi_{\beta_{1}(\mathfrak{x})}, \varpi_{\beta_{2}(\mathfrak{x})}\right\}}, \\
& \left.\min \left\{\gamma_{1}(\mathfrak{x}), \gamma_{2}(\mathfrak{x})\right\} e^{i 2 \pi \min \left\{\varpi_{\gamma_{1}(\mathfrak{x})}, \varpi_{\gamma_{2}(\mathfrak{x})}\right\}}\right): \\
& \mathfrak{x} \in \mathfrak{X}\} .
\end{aligned}
$$

4.

$$
\begin{aligned}
\digamma_{1} \cap \digamma_{2}= & \left\{\left(\mathfrak{x}, \min \left\{\alpha_{1}(\mathfrak{x}), \alpha_{2}(\mathfrak{x})\right\} e^{i 2 \pi \min \left\{\varpi_{\alpha_{1}(\mathfrak{x})}, \varpi_{\alpha_{2}(\mathfrak{x})}\right\}}\right.\right. \\
& \min \left\{\beta_{1}(\mathfrak{x}), \beta_{2}(\mathfrak{x})\right\} e^{i 2 \pi \min \left\{\varpi_{\beta_{1}(\mathfrak{x})}, \varpi_{\left.\beta_{2}(\mathfrak{x})\right\}},\right.} \\
& \max \left\{\gamma_{1}(\mathfrak{x}), \gamma_{2}(\mathfrak{x})\right\} e^{i 2 \pi \max \left\{\varpi_{\gamma_{1}(\mathfrak{x})}, \varpi_{\left.\gamma_{2}(\mathfrak{x})\right\}}\right):} \\
& \mathfrak{x} \in \mathfrak{X}\} .
\end{aligned}
$$

Example 1 Let us consider CTSFSs $\digamma_{1}$ and $\digamma_{2}$ over universal set $\mathfrak{X}=\left\{\mathfrak{x}_{1}, \mathfrak{x}_{2}, \mathfrak{x}_{3}\right\}$ given as follows:

$$
\begin{aligned}
\digamma_{1}= & \left\{\left(\mathfrak{x}_{1}, 0.8 e^{i 2 \pi 0.81}, 0.5 e^{i .2 \pi 0.52}, 0.9 e^{i 2 \pi 0.93}\right),\right. \\
& \left(\mathfrak{x}_{2}, 0.7 e^{i 2 \pi 0.72}, 0.7 e^{i 2 \pi 0.73}, 0.9 e^{i 2 \pi 0.92}\right), \\
& \left.\left(\mathfrak{x}_{3}, 0.8 e^{i 2 \pi 0.82}, 0.7 e^{i 2 \pi 0.71}, 0.9 e^{i 2 \pi 0.91}\right)\right\}
\end{aligned}
$$

and

$$
\begin{aligned}
\digamma_{2}= & \left\{\left(\mathfrak{x}_{1}, 0.9 e^{i 2 \pi 0.91}, 0.6 e^{i 2 \pi 0.62}, 0.8 e^{i 2 \pi 0.83}\right),\right. \\
& \left(\mathfrak{x}_{2}, 0.8 e^{i 2 \pi 0.81}, 0.8 e^{i 2 \pi 0.82}, 0.7 e^{i 2 \pi 0.72}\right), \\
& \left.\left(\mathfrak{x}_{3}, 0.9 e^{i 2 \pi 0.91}, 0.8 e^{i 2 \pi 0.81}, 0.7 e^{i .2 \pi 0.71}\right)\right\}
\end{aligned}
$$

Then it is clear that $\digamma_{1} \subseteq \digamma_{2}$. Also,

$$
\begin{aligned}
\left(\digamma_{1}\right)^{c}= & \left\{\left(x_{1}, 0.9 e^{i 2 \pi 0.93}, 0.5 e^{i 2 \pi 0.52}, 0.8 e^{i 2 \pi 0.81}\right),\right. \\
& \left(\mathfrak{x}_{2}, 0.9 e^{i 2 \pi 0.92}, 0.7 e^{i 2 \pi 0.73}, 0.7 e^{i 2 \pi 0.72}\right), \\
& \left.\left(\mathfrak{x}_{3}, 0.9 e^{i 2 \pi 0.91}, 0.7 e^{i 2 \pi 0.71}, 0.8 e^{i 2 \pi 0.82}\right)\right\}, \\
\digamma_{1} \cup \digamma_{2}= & \left\{\left(\mathfrak{x}_{1}, 0.9 e^{i 2 \pi 0.91}, 0.5 e^{i 2 \pi 0.52},\right.\right. \\
& \left.0.8 e^{i 2 \pi 0.81}\right), \\
& \left(\mathfrak{x}_{2}, 0.8 e^{i 2 \pi 0.81}, 0.7 e^{i 2 \pi 0.73}, 0.7 e^{i 2 \pi 0.72}\right), \\
& \left.\left(\mathfrak{x}_{3}, 0.9 e^{i .2 \pi 0.91}, 0.7 e^{i .2 \pi 0.71}, 0.7 e^{i .2 \pi 0.71}\right)\right\},
\end{aligned}
$$

and

$$
\begin{aligned}
\digamma_{1} \cap \digamma_{2}= & \left\{\left(\mathfrak{x}_{1}, 0.8 e^{i 2 \pi 0.81}, 0.5 e^{i 2 \pi 0.52}, 0.9 e^{i 2 \pi 0.91}\right),\right. \\
& \left(\mathfrak{x}_{2}, 0.7 e^{i 2 \pi 0.72}, 0.7 e^{i 2 \pi 0.73}, 0.7 e^{i 2 \pi 0.71}\right), \\
& \left.\left(\mathfrak{x}_{3}, 0.8 e^{i 2 \pi 0.82}, 0.9 e^{i 2 \pi 0.91}, 0.9 e^{i 2 \pi 0.91}\right)\right\} .
\end{aligned}
$$

\section{Dombi operations of complex T-spherical fuzzy numbers}

In this section, we remind the definitions of Dombi t-norm (TN) and t-conorm (TCN) defined in [60] and we define the arithmetic operations of CTSFNs using Dombi TN and TCN.

Definition 11 [60] Let $f$ and $g$ be two real numbers. Then Dombi TN and Dombi TCN are defined by

$$
\frac{1}{1+\left(\left(\frac{1-g}{g}\right)^{\eta}+\left(\frac{1-h}{h}\right)^{\eta}\right)^{\frac{1}{\eta}}}, \quad \eta>0 .
$$

Dombi t-conorm [60] is given by:

$$
1-\frac{1}{1+\left(\left(\frac{g}{1-g}\right)^{\eta}+\left(\frac{h}{1-h}\right)^{\eta}\right)^{\frac{1}{\eta}}}, \quad \eta>0,
$$

respectively. 
Definition 12 Let $\mathfrak{X}$ be a universe and $\digamma_{1}=\left(\alpha_{1} e^{i 2 \pi \varpi_{\alpha_{1}}}, \beta_{1} e^{i 2 \pi \varpi_{\beta_{1}}}, \gamma_{1} e^{i 2 \pi \varpi_{\gamma_{1}}}\right)$ and $\digamma_{2}=\left(\alpha_{2} e^{i 2 \pi \varpi_{\alpha_{2}}}, \beta_{2} e^{i 2 \pi \varpi_{\beta_{2}}}, \gamma_{2} e^{i 2 \pi \varpi_{\gamma_{2}}}\right)$ are two CTSFNs on $\mathfrak{X}$. Then some Dombi operations between $\digamma_{1}$ and $\digamma_{2}$ are given as follows:

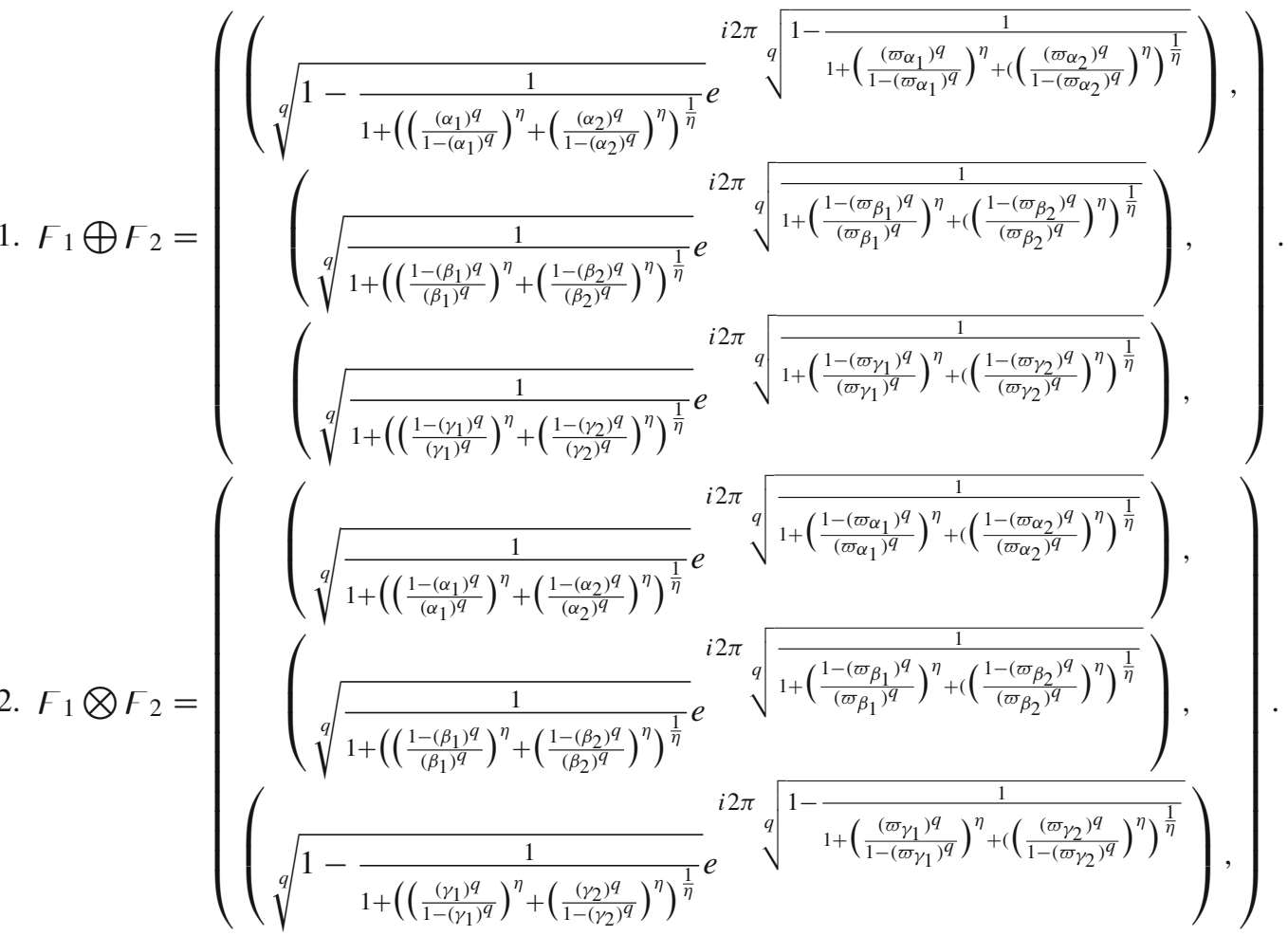

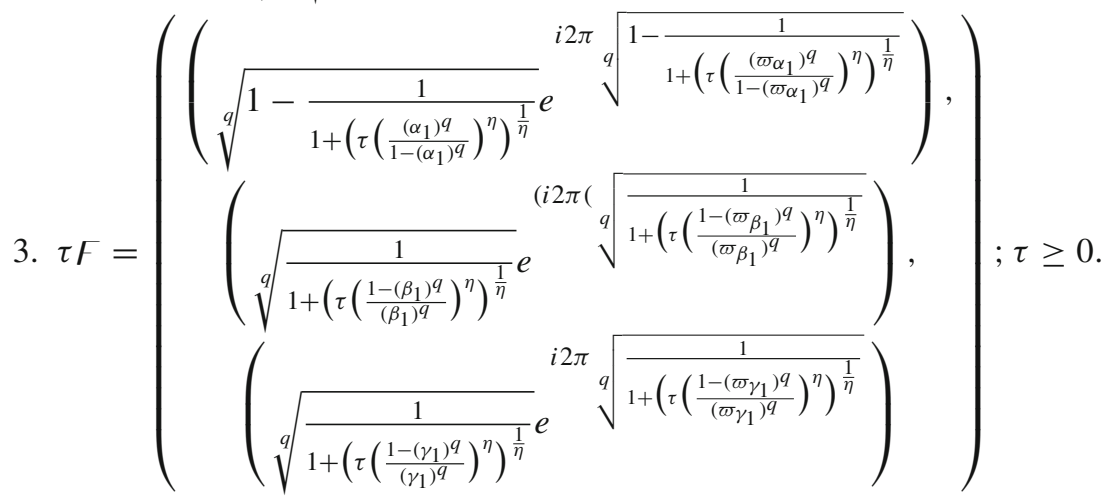

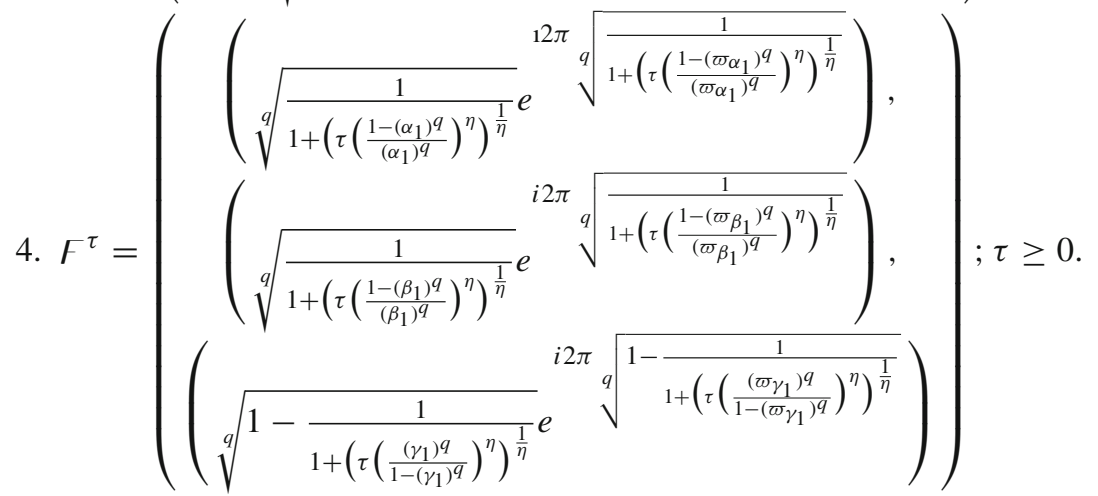


Example 2 Consider two CTSFNs given by

$$
\begin{gathered}
\digamma_{1}=\left(0.8 e^{i 2 \pi 0.81}, 0.5 e^{i 2 \pi 0.52}, 0.9 e^{i 2 \pi 0.93}\right) \\
\digamma_{2}=\left(0.9 e^{i 2 \pi 0.91}, 0.6 e^{i 2 \pi 0.62}, 0.8 e^{i 2 \pi 0.83}\right) .
\end{gathered}
$$

Then for $\eta=1$ and $q=6$

$$
\begin{aligned}
& \digamma_{1} \oplus \digamma_{2}=\left(0.918 e^{i 2 \pi 0.926}, 0.477 e^{i 2 \pi 0.496}, 0.773 e^{i 2 \pi 0.808}\right) \\
& \digamma_{1} \otimes \digamma_{2}=\left(0.773 e^{i 2 \pi 0.784}, 0.477 e^{i 2 \pi 0.496}, 0.918 e^{i 2 \pi 0.942}\right) \\
& \tau \digamma=\left(0.8 e^{i 2 \pi 0.81}, 0.5 e^{i 2 \pi 0.52}, 0.9 e^{i 2 \pi 0.93}\right), \tau=1 \\
& \digamma^{\tau}=\left(0.8 e^{i 2 \pi 0.81}, 0.5 e^{i 2 \pi 0.52}, 0.9 e^{i 2 \pi 0.93}\right) .
\end{aligned}
$$

\section{Dombi weighted aggregation operators of CTSFNs}

In this part, we introduce two operators called complex T-spherical Dombi fuzzy weighted arithmetic averaging

\section{Complex T-spherical Dombi fuzzy weighted arithmetic averaging operator}

Definition 13 Let $\mathfrak{X}$ be a universe and $\digamma_{k}=\left(\alpha_{k} e^{i 2 \pi \omega_{\alpha_{k}}}\right.$, $\left.\beta_{k} e^{i 2 \pi \omega_{\beta_{k}}}, \gamma_{k} e^{i 2 \pi \varpi_{\gamma_{k}}}\right)(k=1,2, \ldots, u)$ be a set of CTSFNs with weight vector $\mathcal{W}=\left(\mathcal{W}_{1}, \mathcal{W}_{2}, \ldots, \mathcal{W}_{u}\right)^{T}$, where $\mathcal{W}_{k}>$ $0, \sum_{k=1}^{u} \mathcal{W}_{k}=1$. Then (CTSDFWAA) operator is defined by a mapping CTSDFWAA : $\digamma^{u} \rightarrow \digamma$, where

$\operatorname{CTSDFWAA}\left(\digamma_{1}, \digamma_{2}, \ldots, \digamma_{u}\right)=\bigoplus_{k=1}^{u} \mathcal{W}_{k} \digamma_{k}$.

Theorem 1 Let $\mathfrak{X}$ be a universe and $\digamma_{k}=\left(\alpha_{k} e^{i 2 \pi \varpi_{\alpha_{k}}}\right.$, $\left.\beta_{k} e^{i 2 \pi \varpi_{\beta_{k}}}, \gamma_{k} e^{i 2 \pi \varpi_{\gamma_{k}}}\right)(k=1,2, \ldots, u)$ be a set of CTSFNs of with weight vector $\mathcal{W}=\left(\mathcal{W}_{1}, \mathcal{W}_{2}, \ldots, \mathcal{W}_{u}\right)^{T}$, where $\mathcal{W}_{\rangle}>0(k=1,2,3, \ldots, u)$ and $\sum_{k=1}^{u} \mathcal{W}_{k}=1$. Then, aggregated value of set using CTSDFWAA is a CTSFN defined as follows:

$\operatorname{CTSDFWAA}\left(\digamma_{1}, \digamma_{2}, \ldots, \digamma_{u}\right)=\bigoplus_{k=1}^{u}\left(\mathcal{W}_{k} \digamma_{k}\right)$

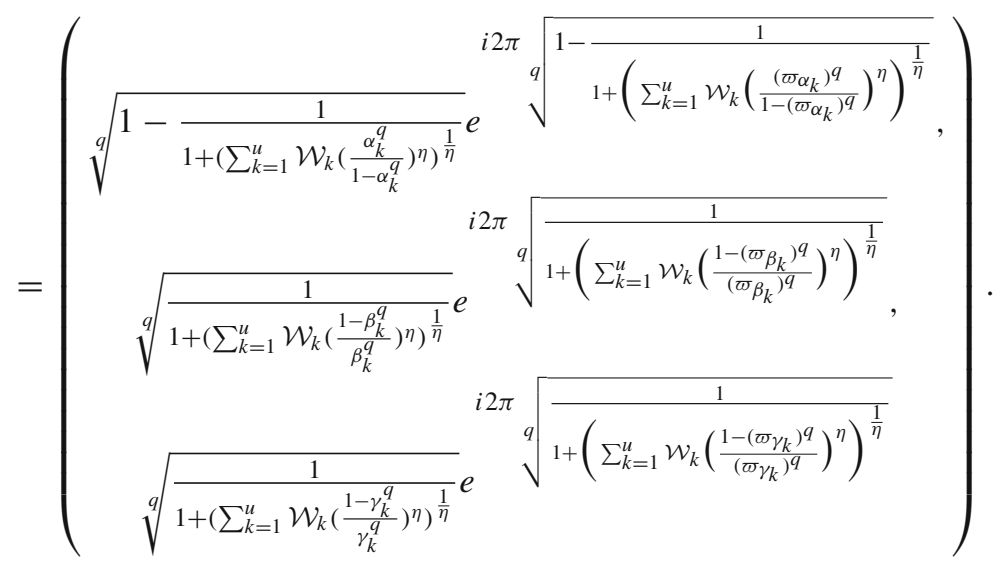

(CTSDFWAA) operator and complex T-spherical Dombi fuzzy weighted geometric averaging (CTSDFWGA) operator. We also obtain some pivotal properties of the introduced operators.
Proof We can simply prove the theory using the mathematical induction method. Using Dombi operations of CTSFNs for $u=2$, we have 


$$
\begin{aligned}
& \operatorname{CTSDFWAA}\left(\digamma_{1}, \digamma_{2}\right)=\mathcal{W}_{1} \digamma_{1} \oplus \mathcal{W}_{2} \digamma_{2}
\end{aligned}
$$

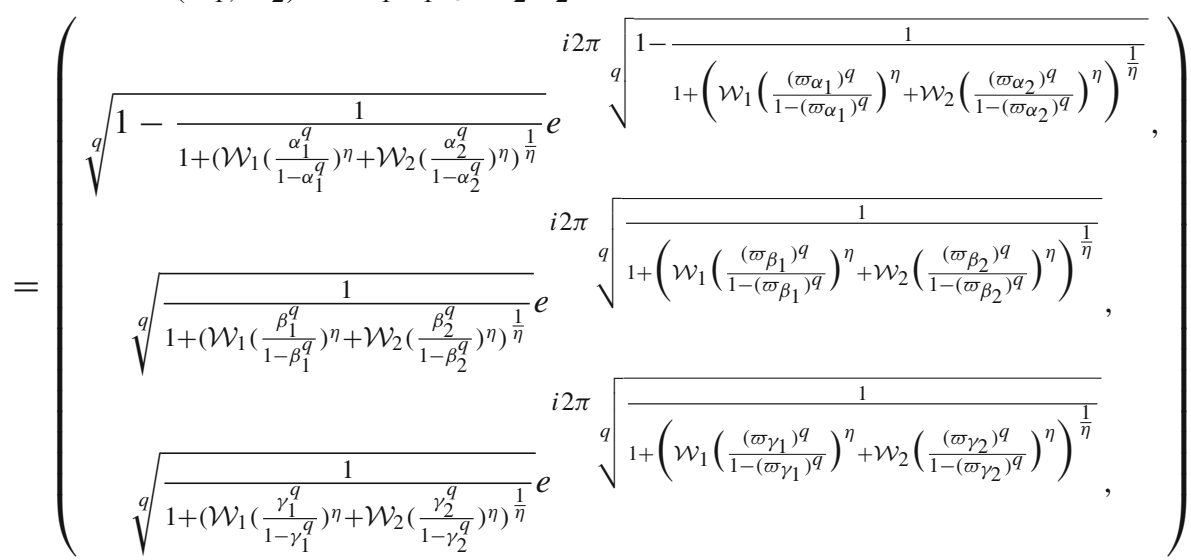

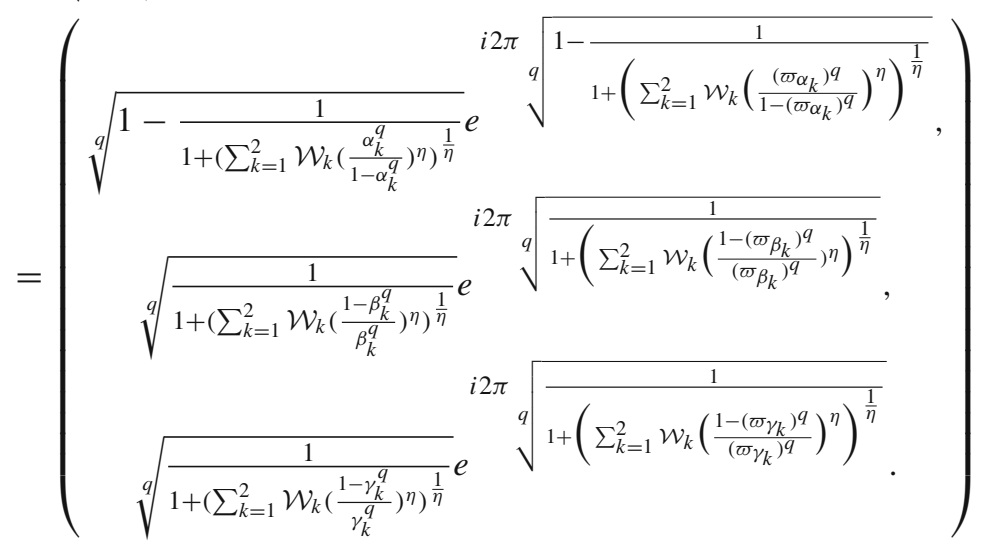

Assume that the equation holds, when $u=\sigma$, i.e.

$$
\begin{aligned}
& \operatorname{CTSDFWAA}\left(\digamma_{1}, \digamma_{2}, \ldots, \digamma_{\sigma}\right)=\mathcal{W}_{1} \digamma_{1} \oplus \mathcal{W}_{2} \digamma_{2} \oplus \ldots \oplus \mathcal{W}_{\sigma} \digamma_{\sigma}
\end{aligned}
$$

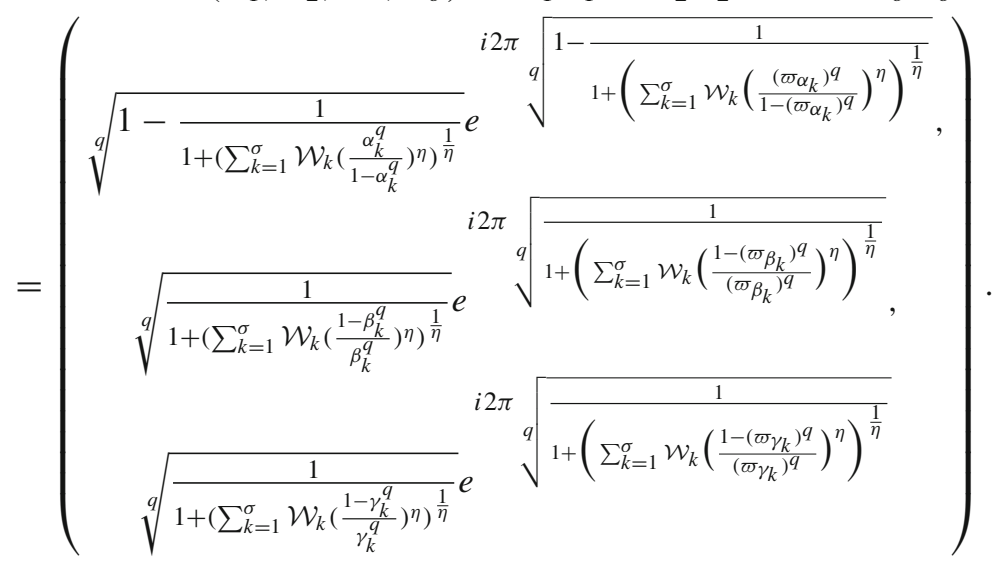


Complex \& Intelligent Systems (2021) 7:2711-2734

2719

If $u=\sigma+1$, then we have

$$
\begin{aligned}
& \operatorname{CTSDFWAA}\left(\digamma_{1}, \digamma_{2}, \ldots, \digamma_{\sigma}\right)=\mathcal{W}_{1} \digamma_{1} \oplus \mathcal{W}_{2} \digamma_{2} \oplus \ldots \oplus \mathcal{W}_{\sigma} \digamma_{\sigma} \oplus \mathcal{W}_{\sigma+1} \digamma_{\sigma+1}
\end{aligned}
$$

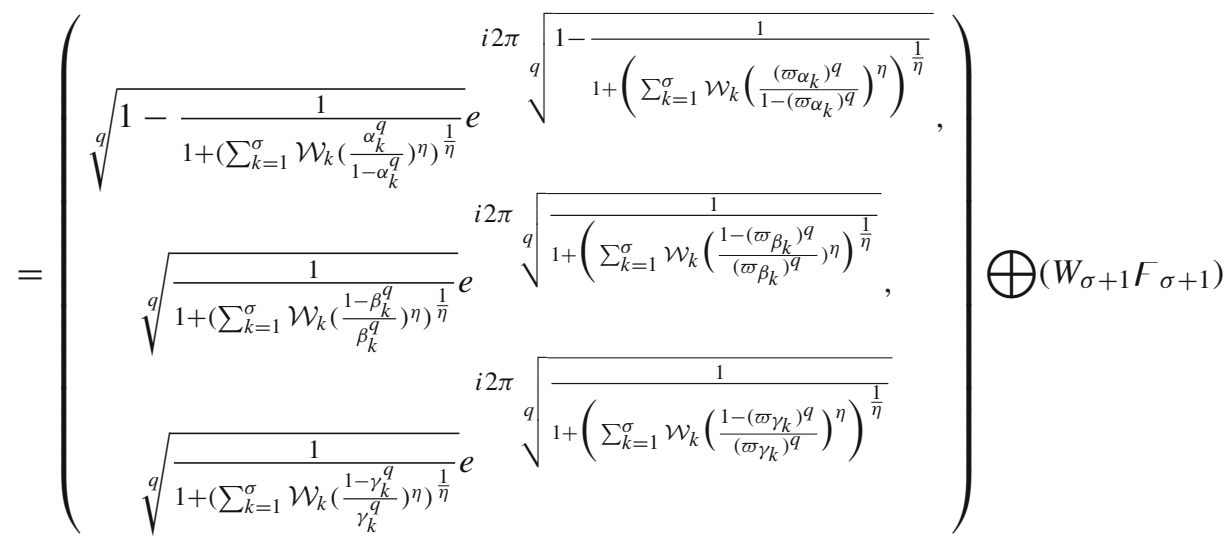

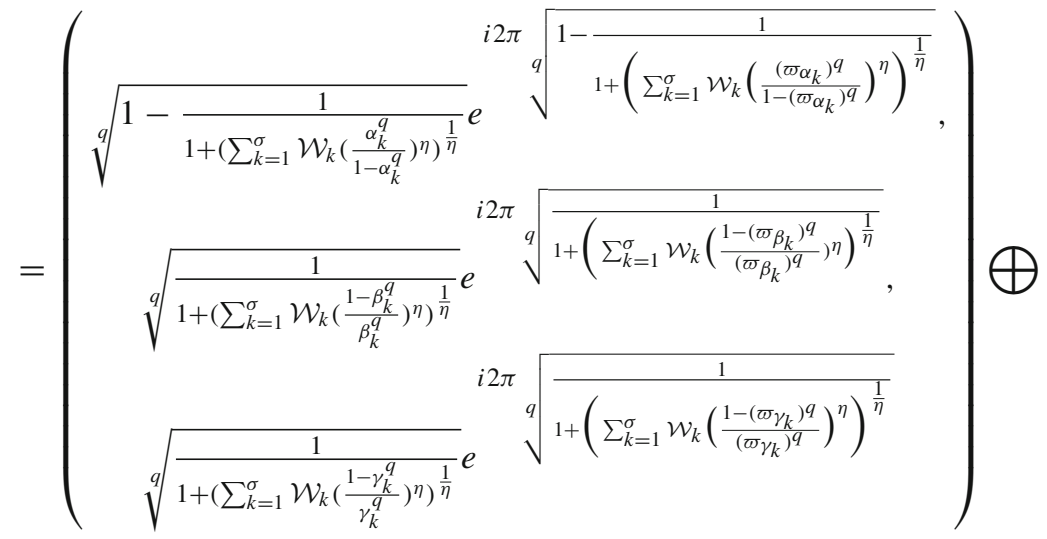

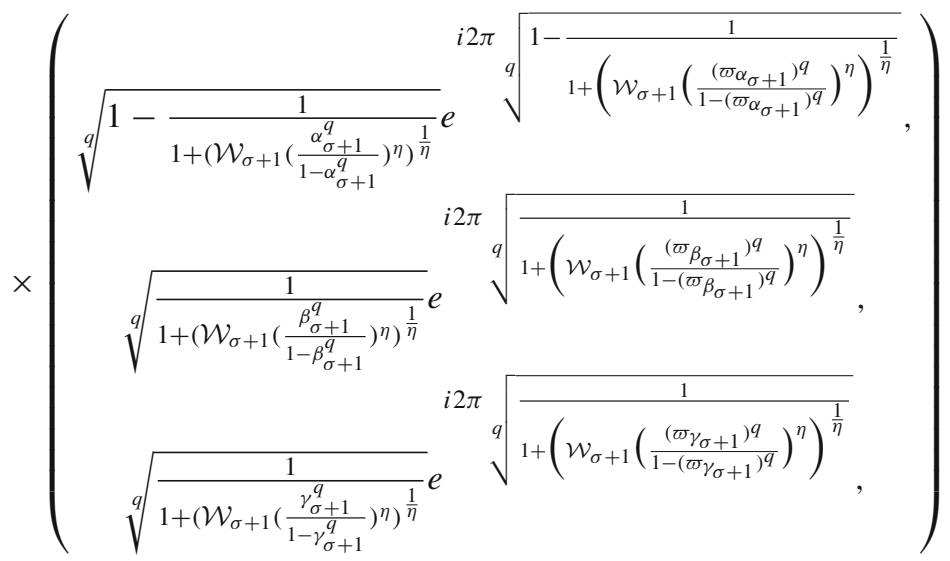

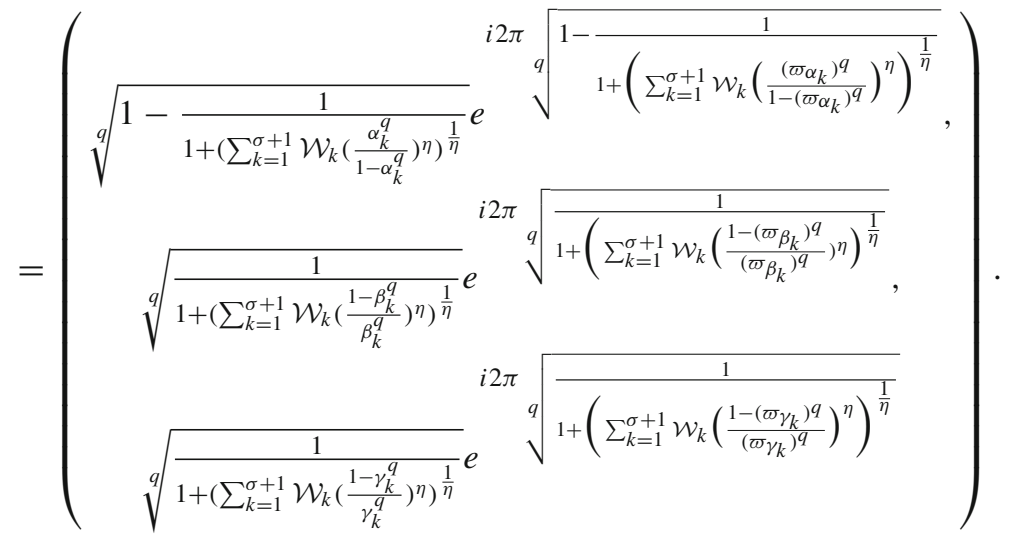

Springer 


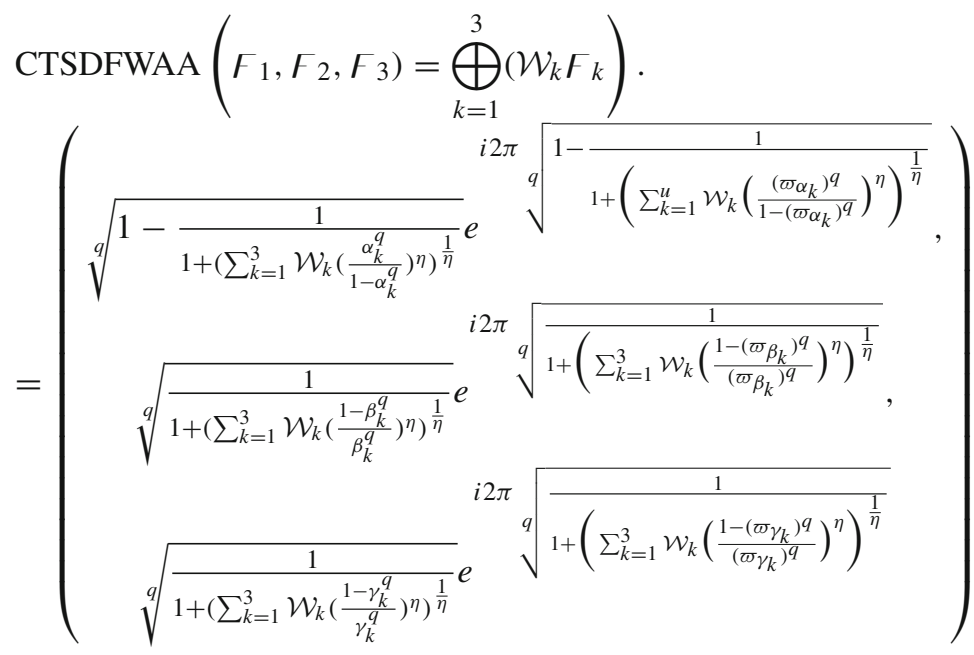

Hence, the theory 3.1 is true for $u=\sigma+1$. Then the equation is true for all $u \in \mathbb{N}$.

Example 3 Consider three CTSFNs with weight vector $\mathrm{W}=$ $(0.5,0.3,0.2)^{\tau}$ and operational parameter $\eta=1$ and $q=6$ given as follows:

$$
\begin{aligned}
& \digamma_{1}=\left(0.8 e^{i 2 \pi 0.81}, 0.5 e^{i 2 \pi 0.52}, 0.9 e^{i 2 \pi 0.93}\right), \\
& \digamma_{2}=\left(0.9 e^{i 2 \pi 0.91}, 0.6 e^{i 2 \pi 0.62}, 0.8 e^{i 2 \pi 0.83}\right), \\
& \digamma_{3}=\left(0.9 e^{i 2 \pi 0.91}, 0.8 e^{i 2 \pi 0.81}, 0.7 e^{i 2 \pi 0.71}\right) .
\end{aligned}
$$

$=\left(0.488 e^{i 2 \pi 0.493}, 0.534 e^{i 2 \pi 0.553}, 0.666 e^{i 2 \pi 0.683}\right)$.

The values when $\eta \neq 1$ are shown in Table 1 .

Theorem 2 (Idempotency)Let $\digamma_{k}=\left(\alpha_{k} e^{i 2 \pi \varpi_{\alpha_{k}}}, \beta_{k} e^{i 2 \pi \varpi_{\beta_{k}}}\right.$, $\left.\gamma_{k} e^{i 2 \pi \varpi_{\gamma_{k}}}\right)(k=1,2, \ldots, u)$ be a set of CTSFNs such that $\digamma_{k}=\digamma$. Then

$\operatorname{CTSDFWAA}\left(\digamma_{1}, \digamma_{2}, \ldots, \digamma_{u}\right)=\digamma$.

Proof Assume that $\digamma_{k}=\digamma$ for all $k=1,2, \ldots, u$. Using Eq. 3, we have

$$
\begin{aligned}
& \operatorname{CTSDFWAA}\left(\digamma_{1}, \digamma_{2}, \ldots, \digamma_{u}\right)=\bigoplus_{k=1}^{u}\left(\mathcal{W}_{k} \digamma_{k}\right)
\end{aligned}
$$

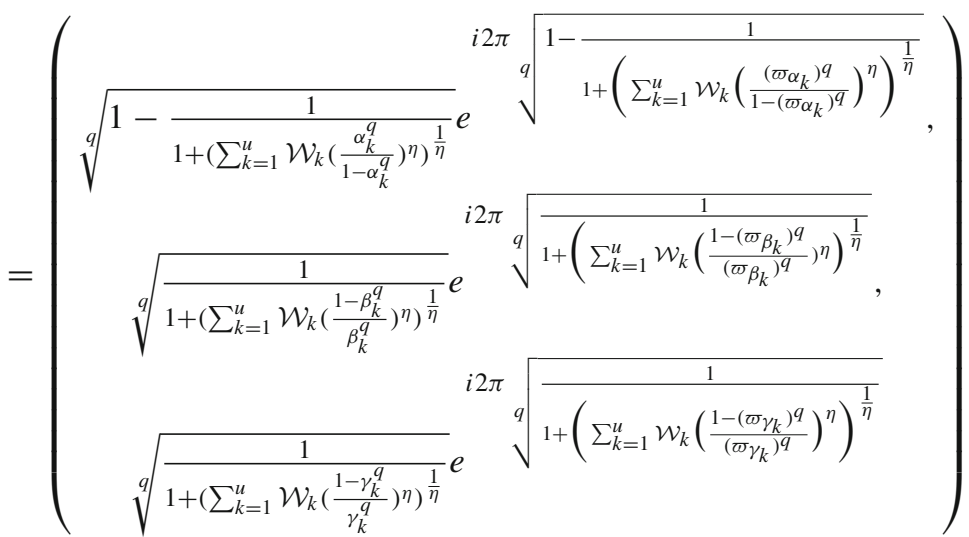

Using the CTSDFWAA operator, we can aggregate the three CTSFNs and find an aggregate value as shown below: 
Table 1 When $\eta \neq 1$

\begin{tabular}{ll}
\hline$\eta$ & CTSFNs \\
\hline 2 & $\left(0.501 e^{i 2 \pi 0.506}, 0.540 e^{i 2 \pi 0.561}, 0.706 e^{i 2 \pi 0.720}\right)$ \\
3 & $\left(0.501 e^{i 2 \pi 0.512}, 0.533 e^{i 2 \pi 0.554}, 0.713 e^{i 2 \pi 0.725}\right)$ \\
4 & $\left(0.513 e^{i 2 \pi 0.518}, 0.526 e^{i 2 \pi 0.548}, 0.714 e^{i 2 \pi 0.726}\right)$ \\
5 & $\left(0.518 e^{i 2 \pi 0.524}, 0.522 e^{i 2 \pi 0.543}, 0.715 e^{i 2 \pi 0.726}\right)$ \\
\hline
\end{tabular}

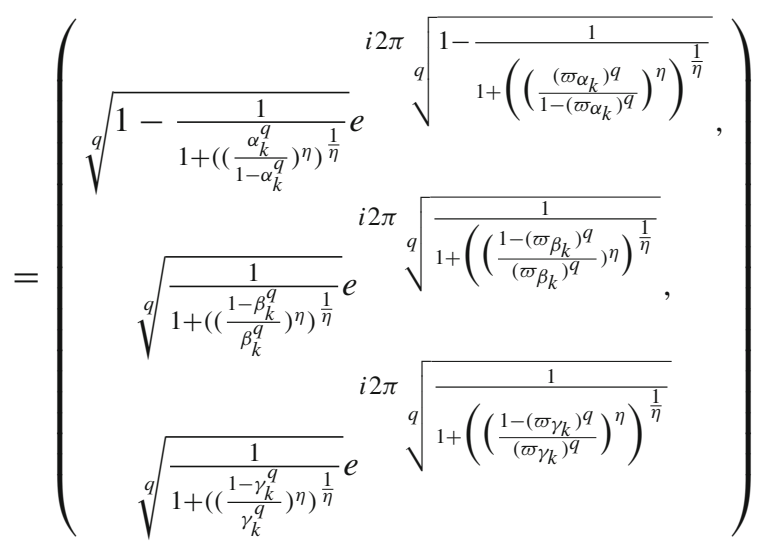

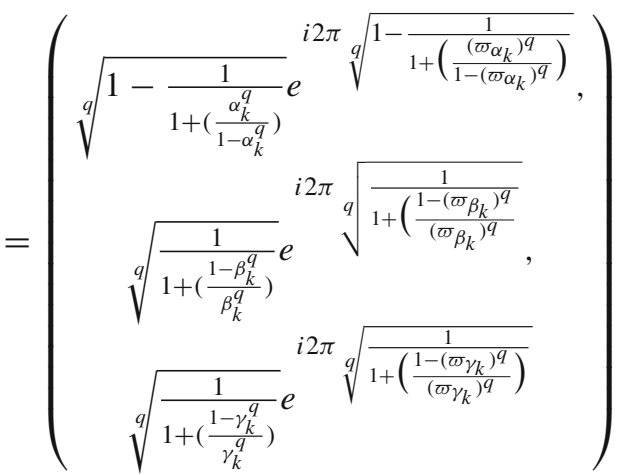

$$
\begin{aligned}
& =\left(\alpha e^{i 2 \pi \varpi_{\alpha}}, \beta e^{i 2 \pi \varpi_{\beta}}, \gamma e^{i 2 \pi \varpi_{\gamma}}\right) \\
& =\digamma \text {. }
\end{aligned}
$$

Theorem 3 (Monotonicity) Let $\digamma_{k}=\left(\alpha_{k} e^{i 2 \pi \varpi_{\alpha_{k}}}\right.$, $\left.\beta_{k} e^{i 2 \pi \varpi_{\beta_{k}}}, \gamma_{k} e^{i 2 \pi \varpi_{\gamma_{k}}}\right)$ and $\digamma_{k}^{\prime}=\left(\alpha_{k}^{\prime} e^{i 2 \pi \varpi_{\alpha_{k}^{\prime}}}\right.$, $\left.\beta_{k}^{\prime} e^{i 2 \pi \varpi_{\beta_{k}^{\prime}}}, \gamma_{k}^{\prime} e^{i 2 \pi \varpi_{\gamma_{k}^{\prime}}}\right)(k=1,2, \ldots, u)$ be two sets of CTSFNs. If $\alpha_{k} \leq \alpha_{k}^{\prime}, \beta_{k} \geq \beta_{k}^{\prime}, \gamma_{k} \geq \gamma_{k}^{\prime}, \varpi_{\alpha_{k}} \leq \varpi_{\alpha_{k}^{\prime}}, \varpi_{\beta_{k}} \geq$ $\varpi_{\beta_{k}^{\prime}}$, and $\varpi_{\gamma_{k}} \geq \varpi_{\gamma_{k}^{\prime}}$ for all $k=1,2, \ldots, u$. Then

$$
\begin{aligned}
& \operatorname{CTSDFWAA}\left(\digamma_{1}, \digamma_{2}, \ldots, \digamma_{u}\right) \\
& \leq \operatorname{CTSDFWAA}\left(\digamma_{1}^{\prime}, \digamma_{2}^{\prime}, \ldots, \digamma_{u}^{\prime}\right) .
\end{aligned}
$$

Proof Let CTSDFWAA $\digamma_{k}=\left(\alpha_{k} e^{i 2 \pi \varpi_{\alpha_{k}}}, \beta_{k} e^{i 2 \pi \varpi_{\beta_{k}}}\right.$, $\left.\gamma_{k} e^{i 2 \pi \varpi_{\gamma_{k}}}\right)$ and

$$
\operatorname{CTSDFWAA} \digamma_{k}^{\prime}=\left(\alpha_{k}^{\prime} e^{i 2 \pi \varpi_{\alpha_{k}^{\prime}}}, \beta_{k}^{\prime} e^{i 2 \pi \varpi_{\beta_{k}^{\prime}}}, \gamma_{k}^{\prime} e^{i 2 \pi \varpi_{\gamma_{k}^{\prime}}}\right) \text {, }
$$

$k=\{1,2,3, \ldots, u\}$ since $\alpha_{\digamma_{1}} \leq \alpha_{\digamma_{1}}, \frac{\alpha_{\digamma_{1}}^{q}}{1-\alpha_{\digamma_{1}}^{q}} \leq \frac{\alpha_{\digamma_{1}}^{q}}{1-\alpha_{\digamma_{1}}^{q}}$. We show first that $\alpha \leq \dot{\alpha}$. So, we have

$$
\begin{aligned}
& \left(\sum_{k=1}^{u} \mathcal{W}_{k}\left(\frac{\alpha_{\digamma_{1}}^{q}}{1-\alpha_{\digamma_{1}}^{q}}\right)^{\eta}\right)^{\frac{1}{\eta}} \\
& \leq\left(\sum_{k=1}^{u} \mathcal{W}_{k}\left(\frac{\alpha_{\check{\digamma}_{1}}^{q}}{1-\alpha_{\check{\digamma}_{1}}^{q}}\right)^{\eta}\right)^{\frac{1}{\eta}} \\
& 1+\left(\sum_{k=1}^{u} \mathcal{W}_{k}\left(\frac{\alpha_{\digamma_{1}}^{q}}{1-\alpha_{\digamma_{1}}^{q}}\right)^{\eta}\right)^{\frac{1}{\eta}} \\
& \leq 1+\left(\sum_{k=1}^{u} \mathcal{W}_{k}\left(\frac{\alpha_{\check{\digamma}_{1}}^{q}}{1-\alpha_{\check{\digamma}_{1}}^{q}}\right)^{\eta}\right)^{\frac{1}{\eta}} \\
& 1 \\
& \overline{1+\left(\sum_{k=1}^{u} \mathcal{W}_{k}\left(\frac{\alpha_{\digamma_{1}}^{q}}{1-\alpha_{\digamma_{1}}^{q}}\right)^{\eta}\right)^{\frac{1}{\eta}}} \\
& \geq \frac{1}{1+\left(\sum_{k=1}^{u} \mathcal{W}_{k}\left(\frac{\alpha_{\digamma_{1}}^{q}}{1-\alpha_{\digamma_{1}}^{q}}\right)^{\eta}\right)^{\frac{1}{\eta}}} \\
& 1-\frac{1}{1+\left(\sum_{k=1}^{u} \mathcal{W}_{k}\left(\frac{\alpha_{\digamma_{1}}^{q}}{1-\alpha_{\digamma_{1}}^{q}}\right)^{\eta}\right)^{\frac{1}{\eta}}} \\
& \leq 1-\frac{1}{1+\left(\sum_{k=1}^{u} \mathcal{W}_{k}\left(\frac{\alpha_{\digamma_{1}}^{q}}{1-\alpha_{\digamma_{1}}^{q}}\right)^{\eta}\right)^{\frac{1}{\eta}}} \\
& \sqrt[q]{1-\frac{1}{1+\left(\sum_{k=1}^{u} \mathcal{W}_{k}\left(\frac{\alpha_{\digamma_{1}}^{q}}{1-\alpha_{\digamma_{1}}^{q}}\right)^{\eta}\right)^{\frac{1}{\eta}}}} \\
& \leq \sqrt[q]{1-\frac{1}{1+\left(\sum_{k=1}^{u} \mathcal{W}_{k}\left(\frac{\alpha_{\digamma_{1}}^{q}}{1-\alpha_{\digamma_{1}}^{q}}\right)^{\eta}\right)^{\frac{1}{\eta}}}} .
\end{aligned}
$$

Hence, $\alpha \leq \alpha^{\prime}$ Similarly, it is easy to show that $\beta \leq \beta$, $\gamma \leq \dot{\gamma}, \varpi_{\alpha} \leq \varpi_{\alpha}^{\prime}, \varpi_{\beta} \leq \varpi_{\beta}, \varpi_{\gamma} \leq \varpi_{\dot{\gamma}}$. Thus, the proof of the theorem is completed. 
Theorem 4 (Boundedness) Let $\digamma_{k}=\left(\alpha_{k} e^{i 2 \pi \varpi_{\alpha_{k}}}\right.$, $\left.\beta_{k} e^{i 2 \pi \omega_{\beta_{k}}}, \gamma_{k} e^{i 2 \pi \varpi_{\gamma_{k}}}\right),(k=1,2,3, \ldots, u)$ a set of CTSFNs with $\digamma_{\min }=\min \left(\digamma_{1}, \digamma_{2}, \digamma_{3}, \ldots, \digamma_{u}\right)$ and $\digamma_{\max }=$ $\max \left(\digamma_{1}, \digamma_{2}, \digamma_{3}, \ldots, \digamma_{u}\right)$. Then

$\digamma_{\min } \preceq C T S D F W A A\left(\digamma_{1}, \digamma_{2}, \digamma_{3}, \ldots, \digamma_{u}\right) \preceq \digamma_{\max }$.

\section{Proof Let}

$$
\begin{aligned}
\digamma_{\min } & =\min \left(\digamma_{1}, \digamma_{2}, \digamma_{3}, \ldots, \digamma_{u}\right) \\
& =\left(\alpha_{k}^{-} e^{i 2 \pi \varpi_{\alpha_{k}}^{-}}, \beta_{k}^{-} e^{i 2 \pi \varpi_{\beta_{k}}^{-}}, \gamma_{k}^{+} e^{i 2 \pi \varpi_{\gamma_{k}}^{+}}\right)
\end{aligned}
$$

and

$$
\begin{aligned}
& \digamma_{\max }=\max \left(\digamma_{1}, \digamma_{2}, \digamma_{3}, \ldots, \digamma_{u}\right) \\
& =\left(\alpha_{k}^{+} e^{i 2 \pi \varpi_{\alpha_{k}}^{+}}, \beta_{k}^{-} e^{i 2 \pi \varpi_{\beta_{k}}^{-}}, \gamma_{k}^{-} e^{i 2 \pi \varpi_{\gamma_{k}}^{-}}\right) \text {. }
\end{aligned}
$$

Therefore,

$$
\begin{aligned}
\min \left(\alpha_{k}\right)= & \alpha_{k}^{-}, \min \left(\beta_{k}\right)=\beta_{k}^{-}, \min \left(\gamma_{k}\right)=\gamma_{k}^{-}, \max \left(\alpha_{k}\right) \\
= & \alpha_{k}^{+}, \max \left(\beta_{k}\right)=\beta_{k}^{+}, \max \left(\gamma_{k}\right)=\gamma_{k}^{+} \\
\min \left(\varpi_{\alpha_{k}}\right) & =\varpi_{\alpha_{k}}^{-}, \min \left(\varpi_{\beta_{k}}\right)=\varpi_{\beta_{k}}^{-}, \min \left(\varpi_{\gamma_{k}}\right) \\
& =\varpi_{\gamma_{k}}^{-}, \max \left(\varpi_{\alpha_{k}}\right)=\varpi_{\alpha_{k}}^{+}, \max \left(\varpi_{\beta_{k}}\right) \\
& =\varpi_{\beta_{k}}^{+}, \max \left(\varpi_{\gamma_{k}}\right)=\varpi_{\gamma_{k}}^{+} .
\end{aligned}
$$

The inequality for amplitude term of membership grade is given as follows:

$$
\begin{aligned}
& \sqrt[q]{1-\frac{1}{1+\left(\sum_{k=1}^{3} \mathcal{W}_{k}\left(\frac{\alpha_{k}^{-q}}{1-\alpha_{k}^{-q}}\right)^{\eta}\right)^{\frac{1}{\eta}}}} \\
& \leq \sqrt[q]{1-\frac{1}{1+\left(\sum_{k=1}^{3} \mathcal{W}_{k}\left(\frac{\alpha_{k}^{q}}{1-\alpha_{k}^{q}}\right)^{\eta}\right)^{\frac{1}{\eta}}}} \\
& \leq \sqrt[q]{1-\frac{1}{1+\left(\sum_{k=1}^{3} \mathcal{W}_{k}\left(\frac{\alpha_{k}^{+q}}{1-\alpha_{k}^{+q}}\right)^{\eta}\right)^{\frac{1}{\eta}}}} .
\end{aligned}
$$

Similarly, the inequality for phase term of membership grade is given as follows:

$$
\begin{aligned}
& \sqrt[q]{1-\frac{1}{1+\left(\sum_{k=1}^{u} \mathcal{W}_{k}\left(\frac{\left(\varpi_{\alpha_{k}}\right)^{-q}}{1-\left(\varpi_{\alpha_{k}}\right)^{-q}}\right)^{\eta}\right)^{\frac{1}{\eta}}}} \\
& \leq \sqrt[q]{1-\frac{1}{1+\left(\sum_{k=1}^{u} \mathcal{W}_{k}\left(\frac{\left(\varpi_{\alpha_{k}}\right)^{q}}{1-\left(\varpi_{\alpha_{k}}\right)^{q}}\right)^{\eta}\right)^{\frac{1}{\eta}}}} \\
& \leq \sqrt[q]{1-\frac{1}{1+\left(\sum_{k=1}^{u} \mathcal{W}_{k}\left(\frac{\left(\varpi_{\alpha_{k}}\right)^{+q}}{1-\left(\varpi_{\alpha_{k}}\right)^{+q}}\right)^{\eta}\right)^{\frac{1}{\eta}}}}
\end{aligned}
$$

In a similar manner, we can get the results for the amplitude and phase terms of abstinence and non-membership grades. Thus,

$\digamma_{\text {min }} \preceq \operatorname{CTSDFWAA}\left(\digamma_{1}, \digamma_{2}, \digamma_{3}, \ldots, \digamma_{u}\right) \preceq \digamma_{\text {max }}$.

\section{Complex T-spherical Dombi fuzzy weighted geometric averaging operator}

Definition 14 Let $\digamma_{k}=\left(\alpha_{k} e^{i 2 \pi \varpi_{\alpha_{k}}}, \beta_{k} e^{i 2 \pi \varpi_{\beta_{k}}}, \gamma_{k} e^{i 2 \pi \varpi_{\gamma_{k}}}\right)$ $(k=1,2, \ldots, u)$ be a set of CTSFNs of with weight vector $\mathcal{W}=\left(\mathcal{W}_{1}, \mathcal{W}_{2}, \ldots, \mathcal{W}_{u}\right)^{T}$, where $\mathcal{W}_{k}>0, \sum_{k=1}^{u} \mathcal{W}_{k}=$ 1. Then (CTSDFWGA) operator is defined by a mapping CTSDFWGA: $\digamma^{u} \rightarrow \digamma$, where

$\operatorname{CTSDFWGA}\left(\digamma_{1}, \digamma_{2}, \ldots, \digamma_{u}\right)=\bigotimes_{k=1}^{u} \digamma_{k}^{\mathcal{W}_{k}}$

Theorem 5 If $\digamma_{k}=\left(\alpha_{k} e^{i 2 \pi \varpi_{\alpha_{k}}}, \beta_{k} e^{i 2 \pi \omega_{\beta_{k}}}, \gamma_{k} e^{i 2 \pi \varpi_{\gamma_{k}}}\right)$ $(k=1,2, \ldots, u)$ be a set of CTSFNs of with weight vector $\mathcal{W}=\left(\mathcal{W}_{1}, \mathcal{W}_{2}, \ldots, \mathcal{W}_{u}\right)^{T}$, where $\mathcal{W}_{k}>0, \sum_{k=1}^{u} \mathcal{W}_{k}=1$ Then (CTSDFWGA) operator the clumped value of these CTSFNs is again a CTSFN. This clumped value can be obtained by the following formula: 


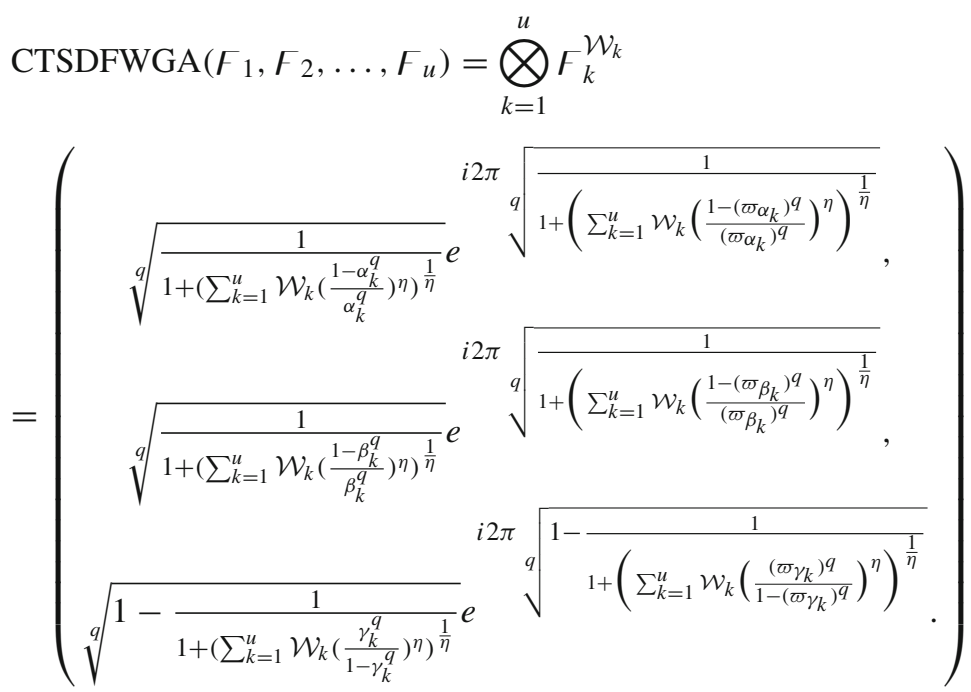

Proof The proof can be made by similar way to the proof of Theorem 9.

Example 4 Let us consider three CTSFNs given as follows:

$$
\begin{gathered}
\digamma_{1}=\left(0.8 e^{i 2 \pi 0.81}, 0.5 e^{i 2 \pi 0.52}, 0.9 e^{i 2 \pi 0.93}\right), \\
\digamma_{2}=\left(0.9 e^{i 2 \pi 0.91}, 0.6 e^{i 2 \pi 0.62}, 0.8 e^{i 2 \pi 0.83}\right), \\
\digamma_{3}=\left(0.9 e^{i 2 \pi 0.91}, 0.8 e^{i 2 \pi 0.81}, 0.7 e^{i 2 \pi 0.71}\right)
\end{gathered}
$$

with the weight vector $\mathbb{W}=(0.5,0.3,0.2)^{\tau}$ and operational parameter $\eta=1$ and $q=6$. Using the CTSDFWGA operator, we can aggregate the three CTSFNs and find a clumped value as given below: $=\left(0.788 e^{i 2 \pi 0.801}, 0.534 e^{i 2 \pi 0.553}, 0.443 e^{i 2 \pi 0.461}\right)$.

The values when $\eta \neq 1$ are shown in Table 2 .

Theorem 6 (Idempotency) Let $\digamma_{k}=\left(\alpha_{k} e^{i 2 \pi \varpi_{\alpha_{k}}}\right.$, $\left.\beta_{k} e^{i 2 \pi \varpi_{\beta_{k}}}, \gamma_{k} e^{i 2 \pi \varpi_{\gamma_{k}}}\right)(k=1,2, \ldots, u)$ be a set of CTSFNs such that $\digamma_{k}=\digamma$. Then

$\operatorname{CTSDFWGA}\left(\digamma_{1}, \digamma_{2}, \ldots, \digamma_{u}\right)=\digamma$.

Proof The proof is similar to the proof of Theorem 2.

$$
\begin{aligned}
& \operatorname{CTSDFWGA}\left(\digamma_{1}, \digamma_{2}, \ldots, \digamma_{u}\right)=\bigotimes_{k=1}^{u} \digamma_{k}^{\mathcal{W}_{k}}
\end{aligned}
$$

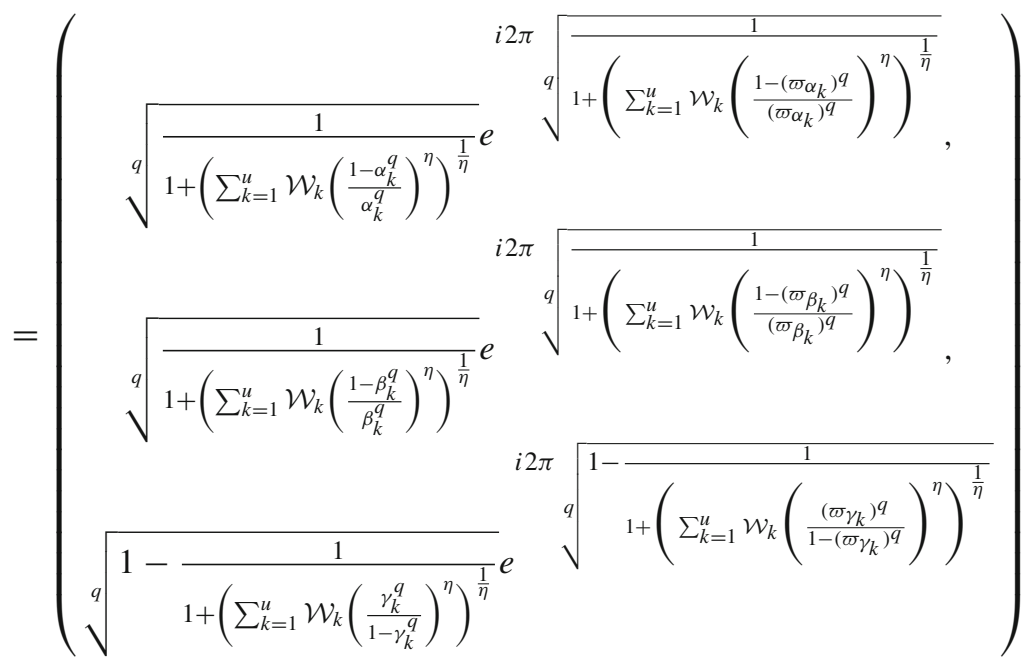


Table 2 When $\eta \neq 1$

\begin{tabular}{ll}
\hline$\eta$ & CTSFNs \\
\hline 2 & $\left(0.855 e^{i 2 \pi 0.869}, 0.540 e^{i 2 \pi 0.561}, 0.476 e^{i 2 \pi 0.505}\right)$ \\
3 & $\left(0.865 e^{i 2 \pi 0.880}, 0.533 e^{i 2 \pi 0.554}, 0.506 e^{i 2 \pi 0.546}\right)$ \\
4 & $\left(0.864 e^{i 2 \pi 0.878}, 0.526 e^{i 2 \pi 0.548}, 0.535 e^{i 2 \pi 0.588}\right)$ \\
5 & $\left(0.861 e^{i 2 \pi 0.875}, 0.522 e^{i 2 \pi 0.543}, 0.875 e^{i 2 \pi 0.629}\right)$ \\
\hline
\end{tabular}

Theorem 7 (Monotonicity) Let $\digamma_{k}=\left(\alpha_{k} e^{i 2 \pi \varpi_{\alpha_{k}}}\right.$, $\left.\beta_{k} e^{i 2 \pi \varpi_{\beta_{k}}}, \gamma_{k} e^{i 2 \pi \varpi_{\gamma_{k}}}\right)$ and $\digamma_{k}^{\prime}=\left(\alpha_{k}^{\prime} e^{i 2 \pi \omega_{\alpha_{k}^{\prime}}}, \beta_{k}^{\prime}\right.$. $\left.e^{i 2 \pi \varpi_{\beta_{k}^{\prime}}}, \gamma_{k}^{\prime} e^{i 2 \pi \omega_{\gamma_{k}^{\prime}}}\right)(k=1,2, \ldots, u)$ be two sets of CTSFNs. If $\alpha_{k} \leq \alpha_{k}^{\prime}, \beta_{k} \geq \beta_{k}^{\prime}, \gamma_{k} \geq \gamma_{k}^{\prime}, \varpi_{\alpha_{k}} \leq \varpi_{\alpha_{k}^{\prime}}, \varpi_{\beta_{k}} \geq$ $\varpi_{\beta_{k}^{\prime}}$, and $\varpi_{\gamma_{k}} \geq \varpi_{\gamma_{k}^{\prime}}$ for all $k$, then

$$
\begin{aligned}
& \operatorname{CTSDFWGA}\left(\digamma_{1}, \digamma_{2}, \ldots, \digamma_{u}\right) \\
& \leq \operatorname{CTSDFWGA}\left(\digamma_{1}^{\prime}, \digamma_{2}^{\prime}, \ldots, \digamma_{u}^{\prime}\right) .
\end{aligned}
$$

Proof The proof can be made by the similar way to Theorem 3.

Theorem 8 (Boundedness) Let $\digamma_{k}=\left(\alpha_{k} e^{i 2 \pi \varpi_{\alpha_{k}}}\right.$, $\left.\beta_{k} e^{i 2 \pi \varpi_{\beta_{k}}}, \gamma_{k} e^{i 2 \pi \varpi_{\gamma_{k}}}\right),(k=1,2,3, \ldots, u)$ be a set of CTSFNs with $\digamma_{\min }=\min \left(\digamma_{1}, \digamma_{2}, \digamma_{3}, \ldots, \digamma_{u}\right)$ and $\digamma_{\max }=\max \left(\digamma_{1}, \digamma_{2}, \digamma_{3}, \ldots, \digamma_{u}\right)$. Then

$\digamma_{\min } \preceq \operatorname{CTSDFWGA}\left(\digamma_{1}, \digamma_{2}, \digamma_{3}, \ldots, \digamma_{u}\right) \preceq \digamma_{\max }$

Proof The proof can be made by the similar way to proof of Theorem 4.

\section{Dombi ordered weighted aggregation operators of CTSFNs}

In this part, we propose two operators, namely, complex T-spherical Dombi fuzzy ordered weighted arithmetic averaging (CTSDFOWAA) operator and complex T-spherical Dombi fuzzy weighted ordered geometric averaging (CTSDFOWGA) operator. Moreover, we also discuss some pivotal properties of these operators.

\section{Complex T-spherical Dombi fuzzy ordered weighted arithmetic averaging operator}

Definition 15 Let $\digamma_{k}=\left(\alpha_{k} e^{i 2 \pi \varpi_{\alpha_{k}}}, \beta_{k} e^{i 2 \pi \varpi_{\beta_{k}}}, \gamma_{k} e^{i 2 \pi \varpi_{\gamma_{k}}}\right)$ $(k=1,2, \ldots, u)$ be a set of CTSFNs of with weight vector $\mathcal{W}=\left(\mathcal{W}_{1}, \mathcal{W}_{2}, \ldots, \mathcal{W}_{u}\right)^{T}$, where $\mathcal{W}_{k}>0, \sum_{k=1}^{u} \mathcal{W}_{k}=1$. Then (CTSDFOWAA) operator is defined by a mapping

CTSDFOWAA : $\digamma^{u} \rightarrow \digamma$, where

$\operatorname{CTSDFOWAA}\left(\digamma_{1}, \digamma_{2}, \ldots, \digamma_{u}\right)=\bigoplus_{k=1}^{u} \mathcal{W}_{k} \digamma_{\sigma(k)}$

and $\left(\sigma_{1}, \sigma_{2}, \ldots, \sigma_{u}\right)$ are the permutations of $\sigma(k)$ having the condition $\left|\digamma_{\sigma(k-1)}\right| \geq\left|\digamma_{\sigma(k)}\right|$ for all $(k=1,2, \ldots, u)$.

Theorem 9 If $\digamma_{k}=\left(\alpha_{k} e^{i 2 \pi \varpi_{\alpha_{k}}}, \beta_{k} e^{i 2 \pi \varpi_{\beta_{k}}}, \gamma_{k} e^{i 2 \pi \varpi_{\gamma_{k}}}\right)$ $(k=1,2, \ldots, u)$ be a set of CTSFNs of with weight vector $\mathcal{W}=\left(\mathcal{W}_{1}, \mathcal{W}_{2}, \ldots, \mathcal{W}_{u}\right)^{T}$, where $\mathcal{W}>0$ and $\sum_{k=1}^{u} \mathcal{W}_{k}=$ 1.

Then aggregated value of set using CTSDFOWAA is a CTSFN defined as follows:

$$
\begin{aligned}
& \operatorname{CTSDFOWAA}\left(\digamma_{1}, \digamma_{2}, \ldots, \digamma_{u}\right)=\bigoplus_{k=1}^{u}\left(\mathcal{W}_{k} \digamma_{\sigma(k)}\right)
\end{aligned}
$$

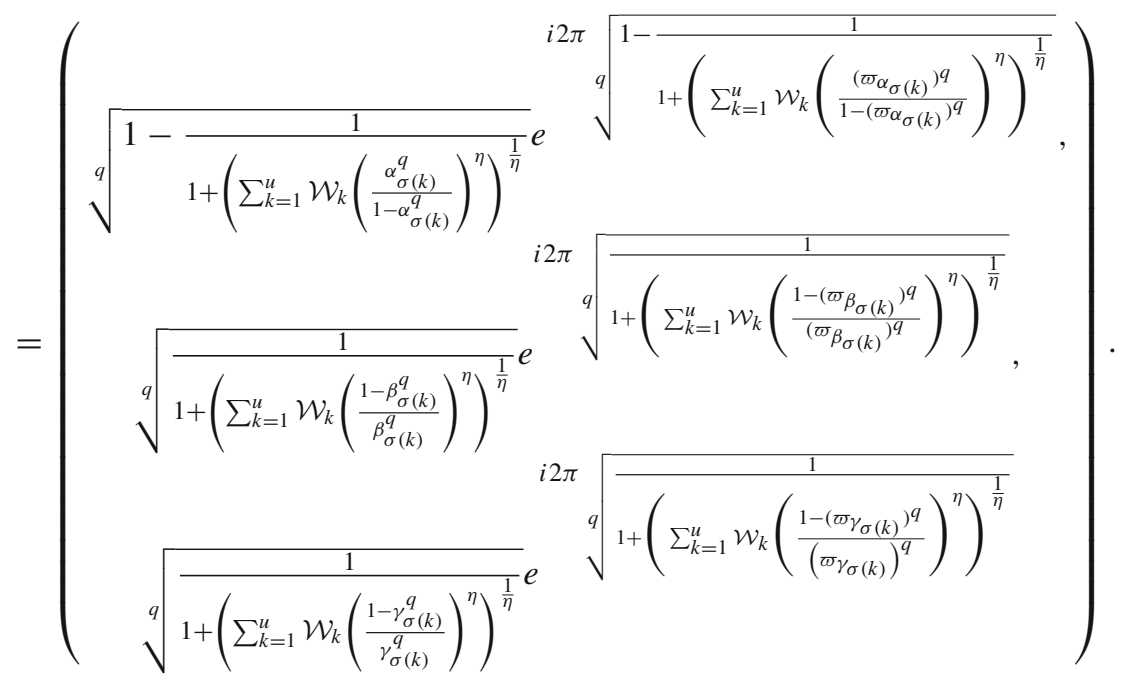


Example 5 Let us consider three CTSFNs given as follows:

$$
\begin{gathered}
\digamma_{1}=\left(0.9 e^{i 2 \pi 0.91}, 0.7 e^{i 2 \pi 0.62}, 0.8 e^{i 2 \pi 0.83}\right), \\
\digamma_{2}=\left(0.9 e^{i 2 \pi 0.91}, 0.8 e^{i 2 \pi 0.81}, 0.3 e^{i 2 \pi 0.50}\right), \\
\digamma_{3}=\left(0.8 e^{i 2 \pi 0.81}, 0.5 e^{i 2 \pi 0.52}, 0.4 e^{i 2 \pi 0.67}\right)
\end{gathered}
$$

having the weight vector $\mathcal{W}=(0.5,0.3,0.2)^{\tau}$ with operational parameter $\eta=1$ and $q=6$. Using the CTSDFOWAA operator, we can aggregate the three CTSFNs. We can find the values of the score function of this CTSFNs

$\Omega\left(\digamma_{1}\right)=0.584, \Omega\left(\digamma_{2}\right)=0.635, \Omega\left(\digamma_{3}\right)=0.604$.

Hence, $\Omega\left(\digamma_{2}\right) \geq \Omega\left(\digamma_{3}\right) \geq \Omega\left(\digamma_{1}\right)$, we will also find that

$$
\begin{gathered}
\digamma_{\sigma 1}=\digamma_{2}=\left(0.9 e^{i 2 \pi 0.91}, 0.8 e^{i 2 \pi 0.81}, 0.3 e^{i 2 \pi 0.50}\right), \\
\digamma_{\sigma 2}=\digamma_{3}=\left(0.8 e^{i 2 \pi 0.81}, 0.5 e^{i 2 \pi 0.52}, 0.4 e^{i 2 \pi 0.67}\right), \\
\digamma_{\sigma 3}=\digamma_{1}=\left(0.9 e^{i 2 \pi 0.91}, 0.7 e^{i 2 \pi 0.62}, 0.8 e^{i 2 \pi 0.83}\right) . \\
\operatorname{CTSDFOWAA}\left(\digamma_{1}, \digamma_{2}, \digamma_{3}\right)=\bigoplus_{k=1}^{3}\left(\mathcal{W}_{k} \digamma_{\sigma(k)}\right)
\end{gathered}
$$

Theorem 11 (Monotonicity) Let $\digamma_{k}=\left(\alpha_{k} e^{i 2 \pi \varpi_{\alpha_{k}}}\right.$, $\left.\beta_{k} e^{i 2 \pi \varpi_{\beta_{k}}}, \gamma_{k} e^{i 2 \pi \varpi_{\gamma_{k}}}\right)$ and $\digamma_{k}^{\prime}=\left(\alpha_{k}^{\prime} e^{i 2 \pi \varpi_{\alpha_{k}^{\prime}}}, \beta_{k}^{\prime}\right.$. $\left.e^{i 2 \pi \varpi_{\beta_{k}^{\prime}}}, \gamma_{k}^{\prime} e^{i 2 \pi \sigma_{\gamma_{k}^{\prime}}}\right)(k=1,2, \ldots, u)$ be two sets of CTSFNs. If $\alpha_{k} \leq \alpha_{k}^{\prime}, \beta_{k} \geq \beta_{k}^{\prime}, \gamma_{k} \geq \gamma_{k}^{\prime}, \varpi_{\alpha_{k}} \leq \varpi_{\alpha_{k}^{\prime}}, \varpi_{\beta_{k}} \geq$ $\varpi_{\beta_{k}^{\prime}}$, and $\varpi_{\gamma_{k}} \geq \varpi_{\gamma_{k}^{\prime}}$ for all $k$. Then

$$
\begin{aligned}
& \operatorname{CTSDFOWAA}\left(\digamma_{1}, \digamma_{2}, \ldots, \digamma_{u}\right) \\
& \quad \preceq \operatorname{CTSDFOWAA}\left(\digamma_{1}^{\prime}, \digamma_{2}^{\prime}, \ldots, \digamma_{u}^{\prime}\right) .
\end{aligned}
$$

Theorem 12 (Boundedness) Let $\digamma_{k}=\left(\alpha_{k} e^{i 2 \pi \omega_{\alpha_{k}}}\right.$, $\left.\beta_{k} e^{i 2 \pi \varpi_{\beta_{k}}}, \gamma_{k} e^{i 2 \pi \varpi_{\gamma_{k}}}\right), k=\{1,2,3, \ldots, u\}$ a set of CTSFNs with $\digamma_{\min }=\min \left(\digamma_{1}, \digamma_{2}, \digamma_{3}, \ldots, \digamma_{u}\right)$ and $\digamma_{\max }=$ $\max \left(\digamma_{1}, \digamma_{2}, \digamma_{3}, \ldots, \digamma_{u}\right)$. Then

$\digamma_{\min } \preceq C T S D F O W A A\left(\digamma_{1}, \digamma_{2}, \digamma_{3}, \ldots, \digamma_{u}\right) \preceq \digamma_{\max }$.

\section{Complex T-spherical Dombi fuzzy ordered weighted geometric averaging operator}

Definition 16 Let $\digamma_{k}=\left(\alpha_{k} e^{i 2 \pi \varpi_{\alpha_{k}}}, \beta_{k} e^{i 2 \pi \varpi_{\beta_{k}}}, \gamma_{k} e^{i 2 \pi \varpi_{\gamma_{k}}}\right)$ $(k=1,2, \ldots, u)$ be a set of CTSFNs of with weight vector

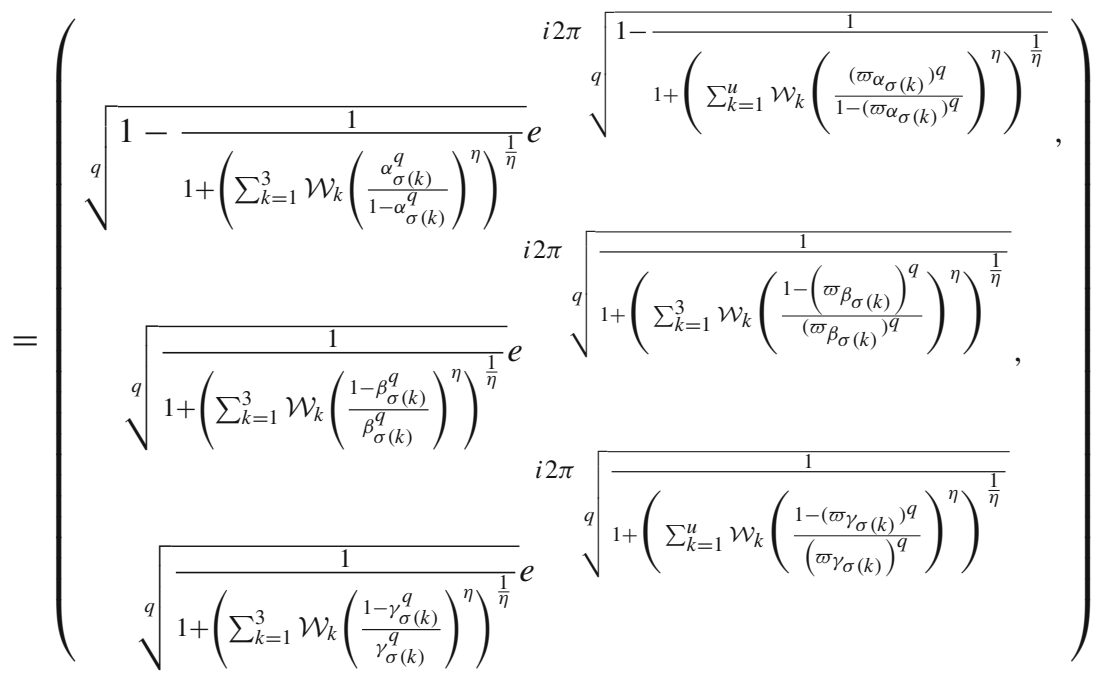

$$
=\left(0.488 e^{i 2 \pi 0.493}, 0.489 e^{i 2 \pi 0.508}, 0.682 e^{i 2 \pi 0.696}\right) .
$$

Theorem 10 (Idempotency) Let $\digamma_{k}=\left(\alpha_{k} e^{i 2 \pi \varpi_{\alpha_{k}}}\right.$, $\left.\beta_{k} e^{i 2 \pi \varpi_{\beta_{k}}}, \gamma_{k} e^{i 2 \pi \varpi_{\gamma_{k}}}\right)(k=1,2, \ldots, u)$ be a set of CTSFNs such that $\digamma_{k}=\digamma$. Then

$\operatorname{CTSDFOWAA}\left(\digamma_{1}, \digamma_{2}, \ldots, \digamma_{u}\right)=\digamma$.
$\mathcal{W}=\left(\mathcal{W}_{1}, \mathcal{W}_{2}, \ldots, \mathcal{W}_{u}\right)^{T}$, where $\mathcal{W}_{k}>0, \sum_{k=1}^{u} \mathcal{W}_{k}=1$ Then (CTSDFOWGA) operator is defined by a mapping CTSDFOWGA : $\digamma_{u} \rightarrow \digamma$, where

$\operatorname{CTSDFOWGA}\left(\digamma_{1}, \digamma_{2}, \ldots, \digamma_{u}\right)=\bigotimes_{k=1}^{u} \digamma_{\sigma(k)}^{\mathcal{W}_{k}}$ 
and $\left(\sigma_{1}, \sigma_{2}, \ldots, \sigma_{u}\right)$ are the permutations of $\sigma(k)$ having the condition $\left|\digamma_{\sigma(k-1)}\right| \geq\left|\digamma_{\sigma(k)}\right|$ for all $(k=1,2, \ldots, u)$

$\operatorname{CTSDFOWGA}\left(\digamma_{1}, \digamma_{2}, \ldots, \digamma_{u}\right)=\bigotimes_{k=1}^{u} \digamma_{\sigma(k)}^{\mathcal{W}_{k}}$
Theorem 13 If $\digamma_{k}=\left(\alpha_{k} e^{i 2 \pi \varpi_{\alpha_{k}}}, \beta_{k} e^{i 2 \pi \varpi_{\beta_{k}}}, \gamma_{k} e^{i 2 \pi \varpi_{\gamma_{k}}}\right)$ $(k=1,2, \ldots, u)$ be a set of CTSFNs of with weight vector $\mathcal{W}=\left(\mathcal{W}_{1}, \mathcal{W}_{2}, \ldots, \mathcal{W}_{u}\right)^{T}$, where $\mathcal{W}_{k}>0, \sum_{k=1}^{u} \mathcal{W}_{k}=$ 1. Then (CTSDFWGA) operator the clumped value of these CTSFNs is again a CTSFN. This clumped value can be obtained by the following formula:

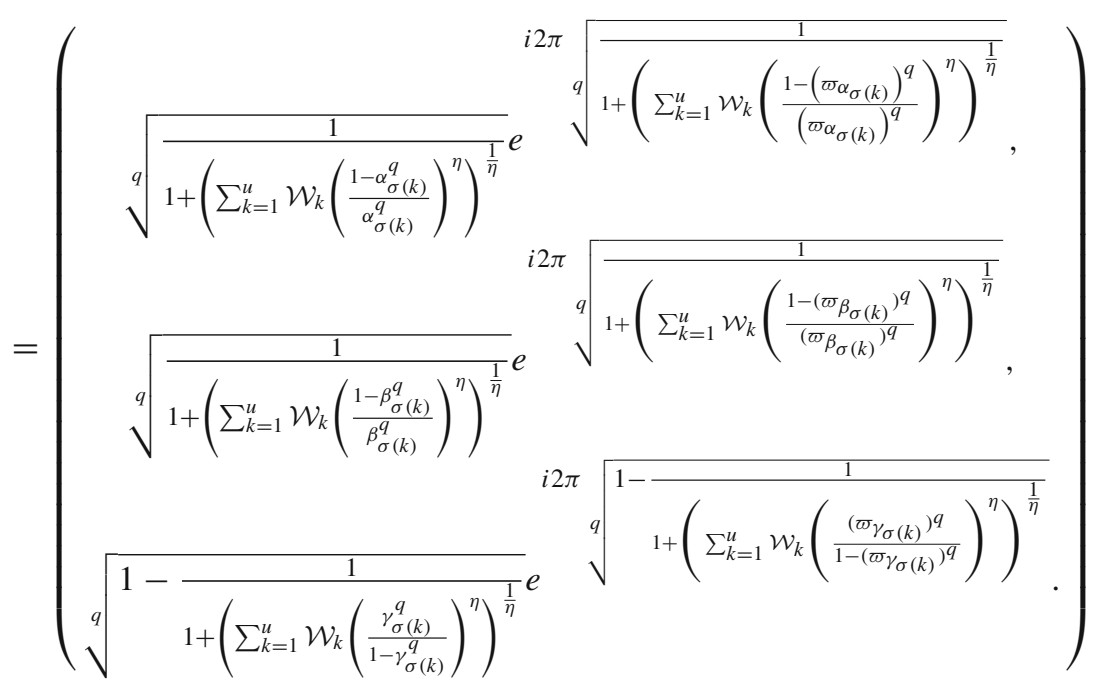

Example 6 Consider CTSFNs given in Example 3.4. Then

$\operatorname{CTSDFOWGA}\left(\digamma_{1}, \digamma_{2}, \digamma_{3}\right)=\bigotimes_{k=1}^{3} \digamma_{\sigma(k)}^{\mathcal{W}_{k}}$

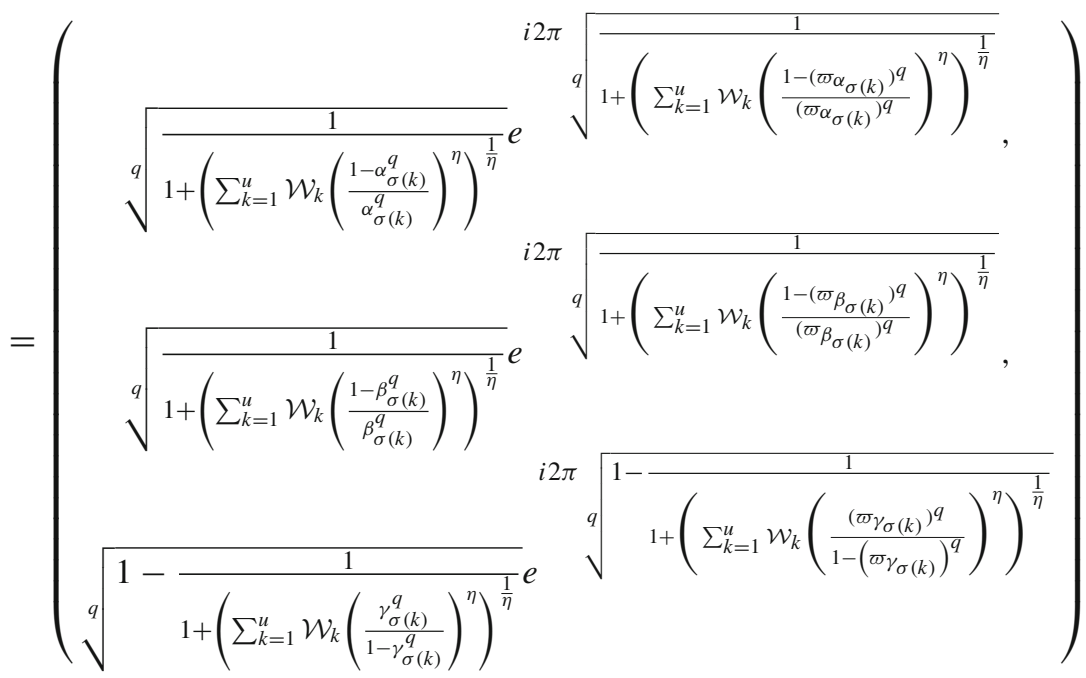


$=\left(0.761 e^{i 2 \pi 0.773}, 0.489 e^{i 2 \pi 0.508}, 0.450 e^{i 2 \pi 0.468}\right)$.

Theorem 14 (Idempotency) Let $\digamma_{k}=\left(\alpha_{k} e^{i 2 \pi \varpi_{\alpha_{k}}}\right.$, $\left.\beta_{k} e^{i 2 \pi \varpi_{\beta_{k}}}, \gamma_{k} e^{i 2 \pi \varpi_{\gamma_{k}}}\right)(k=1,2, \ldots, u)$ be a set of CTSFNs such that $\digamma_{k}=\digamma$. Then

$\operatorname{CTSDFOWGA}\left(\digamma_{1}, \digamma_{2}, \ldots, \digamma_{u}\right)=\digamma$.

Theorem 15 (Monotonicity) Let $\digamma_{k}=\left(\alpha_{k} e^{i 2 \pi \varpi_{\alpha_{k}}}\right.$, $\left.\beta_{k} e^{i 2 \pi \omega_{\beta_{k}}}, \gamma_{k} e^{i 2 \pi \varpi_{\gamma_{k}}}\right)$ and $\digamma_{k}^{\prime}=\left(\alpha_{k}^{\prime} e^{i 2 \pi \omega_{\alpha_{k}^{\prime}}}, \beta_{k}^{\prime} e^{i 2 \pi \varpi_{\beta_{k}^{\prime}}}\right.$, $\left.\gamma_{k}^{\prime} e^{i 2 \pi \omega_{\gamma_{k}^{\prime}}}\right)(k=1,2, \ldots, u)$ be two sets of CTSFNs. If $\alpha_{k} \leq \alpha_{k}^{\prime}, \beta_{k} \geq \beta_{k}^{\prime}, \gamma_{k} \geq \gamma_{k}^{\prime}, \varpi_{\alpha_{k}} \leq \varpi_{\alpha_{k}^{\prime}}, \varpi_{\beta_{k}} \geq \varpi_{\beta_{k}^{\prime}}$, and $\varpi_{\gamma_{k}} \geq \varpi_{\gamma_{k}^{\prime}}$ for all $k$. Then

$$
\begin{aligned}
& \operatorname{CTSDFOWGA}\left(\digamma_{1}, \digamma_{2}, \ldots, \digamma_{u}\right) \\
& \leq \operatorname{CTSDFOWGA}\left(\digamma_{1}^{\prime}, \digamma_{2}^{\prime}, \ldots, \digamma_{u}^{\prime}\right) .
\end{aligned}
$$

Theorem 16 (Boundedness) Let $\digamma_{k}=\left(\alpha_{k} e^{i 2 \pi \varpi_{\alpha_{k}}}\right.$, $\left.\beta_{k} e^{i 2 \pi \varpi_{\beta_{k}}}, \gamma_{k} e^{i 2 \pi \varpi_{\gamma_{k}}}\right),(k=1,2,3, \ldots, u)$ a set of CTSFNs with $\digamma_{\min }=\min \left(\digamma_{1}, \digamma_{2}, \digamma_{3}, \ldots, \ldots, \digamma_{u}\right)$ and $\digamma_{\max }=$ $\max \left(\digamma_{1}, \digamma_{2}, \digamma_{3}, \ldots, \digamma_{u}\right)$. Then

$\digamma_{\text {min }} \preceq \operatorname{CTSDFOWGA}\left(\digamma_{1}, \digamma_{2}, \digamma_{3}, \ldots, \digamma_{u}\right) \preceq \digamma_{\max }$.

\section{MCDM method under CTSF environment}

In this section, we present an MCDM method under CT SF environment.

Let $\kappa=\left\{\kappa_{1}, \kappa_{2}, \ldots, \kappa_{l}\right\}$ be set of alternatives, $\epsilon=$ $\left\{\epsilon_{1}, \epsilon_{2}, \ldots, \epsilon_{s}\right\}$ be a set of criteria. Let us consider $\mathcal{W}=$ $\left(\mathcal{W}_{1}, \mathcal{W}_{2}, \ldots, \mathcal{W}_{s}\right)$ such that $\mathcal{W}_{j} \in(0,1]$ and $\sum_{j=1}^{s} \mathcal{W}_{j}=$ 1 as the weight vector of the criteria which is determined by decision-makers. The steps of the MCDM method are given as follows:

Step 1: The evaluation of the alternative $\kappa_{i}$ according to criteria $\epsilon_{j}$ performed by decision-maker. It can be written as $\zeta_{y j}(j=1,2, \ldots, s ; y=1,2, \ldots, t)$. Hence, CTSFdecision matrix $\mathcal{D} \mathcal{M}=\left[\zeta_{y j}\right]_{t \times s}$ can be constructed as follows:

$$
\mathcal{D} \mathcal{M}=\left[\zeta_{y j}\right]_{t \times s}=\left(\begin{array}{cccc}
\zeta_{11} & \zeta_{12} & \cdots & \zeta_{1 s} \\
\zeta_{21} & \zeta_{22} & \cdots & \zeta_{2 s} \\
\vdots & \vdots & \cdots & \vdots \\
\zeta_{t 1} & \zeta_{t 2} & \cdots & \zeta_{t s}
\end{array}\right)
$$

Here $\zeta_{y j}=\left(\alpha_{y j} e^{i 2 \pi \varpi_{y j}}, \beta_{y j} e^{i 2 \pi \varpi_{y j}}, \gamma_{y j} e^{i 2 \pi \varpi_{y j}}\right)$.

Step 2: Find the aggregated value denoted by $\mathbb{A}_{y}(y=$ $1,2,3, \ldots, l)$ using the $C T S D F W A A$ operators.

Step 3: Find the score values, for each $\mathbb{A}_{y} y=1,2,3, \ldots, l$

Step 4: Choose the alternative which has a maximum score value.

\section{Application}

The COVID-19 outbreak first appeared in Wuhan city of China in December 2019 and spread rapidly all over the world [70,71]. Until May 5, 2021, 153,790,183 people were infected with the COVID-19 and 3,218,080 people died [72]. Also, the spread of COVID-19 still continuous. In some papers, mathematical analysis revealing the spread of such a deathly disease have been presented [73-75].

In this section, an application of the proposed method to determine a patient infected by COVID-19 is presented. In this application, after we discuss by infectious diseases physician, we determine the criteria as a basic symptoms of COVID-19. Set of the symptoms is considered as $\epsilon=\left\{\epsilon_{1}=\right.$ Fever, $\epsilon_{2}=$ headache, $\epsilon_{3}=$ dyspnea,$\epsilon_{4}=$ cough $\}$. Also, we consider the five patients $p_{1}, p_{2}, p_{3}, p_{4}$ and $p_{5}$. For each of patients, data measured along with 14 days according to symptoms are given in Tables 3, 4, 5 and 6.

Here we transform data given in tables to CTSFN. We will only explain transforming process of data given in Table 3 . Other transforming of the tables will not be showed.

We establish amplitude terms and phase terms according to fever degree and days, respectively. First, we classify the degree of fever for MD (38.6-39.5), $\mathrm{NeD}(37.6-38.5)$, and NMD (36.5-37.5). Then we assign values from 0.1 to 0.9 for fever intervals. This is shown in Table 7.

For each of MD, NeD and NMD, we find the arithmetic mean of the fever values. Then we assign a value from 0.1 to 0.9 .

These values are shown in Table 8 and Table 9.

We divide the number of MD, NeD and NMD days by 14 for each patient to obtain the phase terms. For example, according to Table 3, we see that fever of $p_{1}$ is in MD for 6 days, in NeD for 5 days and in NMD for 3 days. Then phase terms for $\mathrm{MD}, \mathrm{NeD}$ and $\mathrm{NMD}$ are $\frac{6}{14}=0.43, \frac{5}{14}=0.36$, and $\frac{3}{14}=0.21$. All of phase terms for patients are shown in Table 10.

In a similar way, we can obtain all of the CTSF values for each of the patients with respect to symptoms using following classification tables for other symptoms.

Here we use the steps of the proposed method. 
Table 3 Fever values of patients measured for 14 days

Table 4 Headache values of patients measured for 14 days

Table 5 Dyspnea values of patients measured for 14 days

Table 6 Cough values of patients measured for 14 days

Table 7 Classification of measured fever degrees according to $\mathrm{MD}, \mathrm{NeD}$, and NMD

\begin{tabular}{|c|c|c|c|c|c|c|c|c|c|}
\hline & 1 & 2 & 3 & 4 & 5 & 6 & 7 & 8 & 9 \\
\hline$p_{1}$ & 37.80 & 38.00 & 38.20 & 38.60 & 39.00 & 39.30 & 39.50 & 39.00 & 38.60 \\
\hline$p_{2}$ & 38.10 & 38.50 & 38.80 & 39.10 & 39.40 & 40.00 & 40.10 & 39.50 & 39.20 \\
\hline$p_{3}$ & 37.50 & 38.10 & 38.60 & 38.20 & 38.10 & 38.00 & 37.70 & 37.50 & 37.20 \\
\hline$p_{4}$ & 38.20 & 38.80 & 39.00 & 39.60 & 39.60 & 40.00 & 40.20 & 39.50 & 39.00 \\
\hline$p_{5}$ & 36.80 & 36.60 & 37.30 & 38.00 & 38.30 & 38.80 & 38.10 & 37.70 & 37.20 \\
\hline 10 & & \multicolumn{2}{|c|}{11} & \multicolumn{2}{|c|}{12} & \multicolumn{3}{|c|}{13} & 14 \\
\hline 38.10 & & \multicolumn{2}{|c|}{37.80} & \multicolumn{2}{|c|}{37.50} & \multicolumn{3}{|c|}{37.20} & 37.00 \\
\hline 38.70 & & \multicolumn{2}{|c|}{38.30} & \multicolumn{2}{|c|}{38.00} & \multicolumn{3}{|c|}{37.70} & 37.40 \\
\hline 37.00 & & \multicolumn{2}{|c|}{37.40} & \multicolumn{2}{|c|}{37.10} & \multicolumn{3}{|c|}{36.80} & 36.60 \\
\hline 38.60 & & \multicolumn{2}{|c|}{38.10} & \multicolumn{2}{|c|}{37.70} & \multicolumn{3}{|c|}{37.40} & 37.00 \\
\hline 37.20 & & \multicolumn{2}{|c|}{36.80} & \multicolumn{2}{|c|}{36.60} & \multicolumn{3}{|c|}{36.90} & 36.50 \\
\hline
\end{tabular}

\begin{tabular}{|c|c|c|c|c|c|c|c|c|c|c|c|c|c|c|}
\hline & 1 & 2 & 3 & 4 & 5 & 6 & 7 & 8 & 9 & 10 & 11 & 12 & 13 & 14 \\
\hline$p_{1}$ & 3.7 & 5.5 & 5.8 & 6.2 & 6.8 & 7.7 & 8.2 & 9.3 & 9.4 & 9.5 & 8.6 & 7.4 & 5.6 & 3.4 \\
\hline$p_{2}$ & 9.3 & 9.2 & 9.4 & 8.8 & 8.7 & 8.6 & 9.6 & 6.8 & 6.8 & 6.6 & 5.6 & 4.7 & 3.9 & 3.6 \\
\hline$p_{3}$ & 1.5 & 2.4 & 3.2 & 3.8 & 6.6 & 5.8 & 6.8 & 6.5 & 6.9 & 8.3 & 9.4 & 9.6 & 9.8 & 8.7 \\
\hline$p_{4}$ & 6.7 & 6.8 & 6.3 & 6.6 & 9.6 & 9.7 & 9.5 & 9.4 & 9.7 & 8.9 & 9.1 & 6.2 & 4.3 & 3.8 \\
\hline$p_{5}$ & 3.6 & 3.7 & 3.9 & 5.2 & 5.8 & 6.8 & 6.9 & 6.9 & 7.2 & 8.1 & 6.9 & 6.5 & 5.9 & 3.8 \\
\hline
\end{tabular}

\begin{tabular}{lllllllllllllll}
\hline & 1 & 2 & 3 & 4 & 5 & 6 & 7 & 8 & 9 & 10 & 11 & 12 & 13 & 14 \\
\hline$p_{1}$ & 2.8 & 3.3 & 3.9 & 4.8 & 5.9 & 6.4 & 6.9 & 7.9 & 9.6 & 9.9 & 9.7 & 9.6 & 6.9 & 6.4 \\
$p_{2}$ & 9.8 & 9.9 & 9.5 & 9.3 & 8.4 & 6.9 & 6.5 & 6.2 & 5.6 & 3.9 & 3.8 & 3.5 & 3.4 & 3.6 \\
$p_{3}$ & 1.9 & 2.8 & 3.3 & 3.8 & 3.9 & 6.8 & 7.9 & 8.8 & 9.3 & 6.9 & 6.5 & 5.8 & 5.3 & 4.9 \\
$p_{4}$ & 9.7 & 9.2 & 9.6 & 6.9 & 6.5 & 3.9 & 6.1 & 6.9 & 6.9 & 9.8 & 9.9 & 9.9 & 9.8 & 9.9 \\
$p_{5}$ & 2.2 & 2.5 & 2.7 & 3.1 & 3.9 & 3.8 & 6.7 & 5.4 & 6.9 & 6.6 & 6.9 & 8.9 & 9.8 & 9.8 \\
\hline
\end{tabular}

\begin{tabular}{|c|c|c|c|c|c|c|c|c|c|c|c|c|c|c|}
\hline & 1 & 2 & 3 & 4 & 5 & 6 & 7 & 8 & 9 & 10 & 11 & 12 & 13 & 14 \\
\hline$p_{1}$ & 3.1 & 3.4 & 4.8 & 6.7 & 8.6 & 8.2 & 8.3 & 6.6 & 6.2 & 5.4 & 3.9 & 3.5 & 3.6 & 3.4 \\
\hline$p_{2}$ & 3.9 & 6.1 & 6.3 & 6.8 & 6.9 & 8.8 & 9.7 & 9.8 & 9.9 & 9.8 & 9.8 & 9.9 & 6.9 & 6.8 \\
\hline$p_{3}$ & 2.9 & 3.6 & 3.9 & 5.9 & 6.1 & 6.8 & 6.9 & 6.9 & 9.3 & 9.9 & 9.7 & 9.8 & 9.9 & 9.8 \\
\hline$p_{4}$ & 9.7 & 9.9 & 9.6 & 9.9 & 9.8 & 6.9 & 6.5 & 6.9 & 6.9 & 3.9 & 3.2 & 3.1 & 2.8 & 2.7 \\
\hline$p_{5}$ & 3.8 & 3.9 & 5.5 & 6.7 & 6.8 & 6.9 & 6.7 & 8.8 & 7.8 & 7.9 & 8.9 & 9.3 & 9.8 & 9.8 \\
\hline
\end{tabular}

\begin{tabular}{llllllllll}
\hline & 0.1 & 0.2 & 0.3 & 0.4 & 0.5 & 0.6 & 0.7 & 0.8 & 0.9 \\
\hline MD & 38.6 & 38.7 & 38.8 & 38.9 & 39 & 39.1 & 39.2 & 39.3 & 39.4 \\
NeD & 37.7 & 37.8 & 37.9 & 38 & 38.1 & 38.2 & 38.3 & 38.4 & 38.5 \\
NMD & 36.7 & 36.8 & 36.9 & 37 & 37.1 & 37.2 & 37.3 & 37.4 & 37.5 \\
\hline
\end{tabular}


Table 8 Arithmetic mean (AM) of fever values for $\mathrm{MD}, \mathrm{NeD}$, and NMD

Table $9 \mathrm{MD}, \mathrm{NeD}$, and NMD values in $[0,1]$ for patients

Table 10 Phase terms

\begin{tabular}{llll}
\hline & MD & NeD & NMD \\
\hline$p_{1}$ & 39.00 & 37.98 & 37.23 \\
$p_{2}$ & 39.35 & 38.12 & 37.40 \\
$p_{3}$ & 38.60 & 38.02 & 37.14 \\
$p_{4}$ & 39.37 & 38.00 & 37.20 \\
$p_{5}$ & 38.80 & 38.03 & 36.88 \\
\hline
\end{tabular}

\begin{tabular}{llll}
\hline & MD & NeD & NMD \\
\hline$p_{1}$ & 0.5 & 0.4 & 0.6 \\
$p_{2}$ & 0.9 & 0.5 & 0.8 \\
$p_{3}$ & 0.1 & 0.4 & 0.5 \\
$p_{4}$ & 0.9 & 0.4 & 0.6 \\
$p_{5}$ & 0.3 & 0.4 & 0.3 \\
\hline
\end{tabular}

\begin{tabular}{llll}
\hline & MD & NeD & NMD \\
\hline$p_{1}$ & 0.43 & 0.36 & 0.21 \\
$p_{2}$ & 0.57 & 0.36 & 0.07 \\
$p_{3}$ & 0.07 & 0.36 & 0.57 \\
$p_{4}$ & 0.64 & 0.21 & 0.14 \\
$p_{5}$ & 0.07 & 0.29 & 0.64 \\
\hline
\end{tabular}

Step 1: $\mathcal{D} \mathcal{M}$ matrix is constructed by taking into consideration the above data.
Here we consider $q=9$.

Step 2: Aggregated values are found for each of the patients by applying $C T S D F W A A$ and $C T S D F W G A$ operators to rows of the $\mathcal{M D}$.
Table 11 Classification of measured headache degrees according to $\mathrm{MD}, \mathrm{NeD}$, and NMD

\begin{tabular}{llllllllll}
\hline & 0.1 & 0.2 & 0.3 & 0.4 & 0.5 & 0.6 & 0.7 & 0.8 & 0.9 \\
\hline MD & 7.3 & 7.6 & 7.9 & 8.2 & 8.5 & 8.8 & 9.1 & 9.4 & 9.7 \\
NeD & 4.3 & 4.6 & 4.9 & 5.2 & 5.5 & 5.8 & 6.1 & 6.4 & 6.7 \\
NMD & 1.3 & 1.6 & 1.9 & 2.2 & 2.5 & 2.8 & 3.1 & 3.4 & 3.7 \\
\hline
\end{tabular}


Table 12 Obtaining results for headache using Tables 4 and 11

Table 13 Classification of measured dyspnea degrees NMD

Table 14 Obtaining results for dyspnea using Tables 5 and 13

Table 15 Classification of measured cough degrees according to $\mathrm{MD}, \mathrm{NeD}$, and NMD

Table 16 Obtaining results for cough using Tables 6 and 15 according to $\mathrm{MD}, \mathrm{NeD}$, and

\begin{tabular}{|c|c|c|c|c|c|c|c|c|c|c|c|}
\hline & MD & $\mathrm{NeD}$ & NMD & & MD & $\mathrm{NeD}$ & NMD & & MD & $\mathrm{NeD}$ & NMD \\
\hline$p_{1}$ & 8.59 & 5.98 & 3.55 & $p_{1}$ & 0.5 & 0.7 & 0.8 & $p_{1}$ & 0.50 & 0.36 & 0.14 \\
\hline$p_{2}$ & 9.09 & 6.10 & 3.75 & $p_{2}$ & 0.7 & 0.7 & 0.9 & $p_{2}$ & 0.50 & 0.36 & 0.14 \\
\hline$p_{3}$ & 9.16 & 6.52 & 2.73 & $p_{3}$ & 0.7 & 0.8 & 0.5 & $p_{3}$ & 0.36 & 0.36 & 0.29 \\
\hline$p_{4}$ & 9.41 & 6.15 & 3.80 & $p_{4}$ & 0.8 & 0.7 & 0.9 & $p_{4}$ & 0.50 & 0.43 & 0.07 \\
\hline$p_{5}$ & 7.65 & 6.36 & 3.75 & $p_{5}$ & 0.2 & 0.8 & 0.9 & $p_{5}$ & 0.14 & 0.57 & 0.29 \\
\hline \multicolumn{5}{|c|}{ AM of headache degrees } & \multicolumn{3}{|c|}{$\mathrm{MD}, \mathrm{NeD}$, and NMD values } & \multicolumn{4}{|c|}{ Phase terms } \\
\hline
\end{tabular}

\begin{tabular}{llllllllll}
\hline & 0.1 & 0.2 & 0.3 & 0.4 & 0.5 & 0.6 & 0.7 & 0.8 & 0.9 \\
\hline MD & 7.3 & 7.6 & 7.9 & 8.2 & 8.5 & 8.8 & 9.1 & 9.4 & 9.7 \\
NeD & 4.3 & 4.6 & 4.9 & 5.2 & 5.5 & 5.8 & 6.1 & 6.4 & 6.7 \\
NMD & 1.3 & 1.6 & 1.9 & 2.2 & 2.5 & 2.8 & 3.1 & 3.4 & 3.7 \\
\hline
\end{tabular}

\begin{tabular}{|c|c|c|c|c|c|c|c|c|c|c|c|}
\hline & MD & $\mathrm{NeD}$ & NMD & & MD & $\mathrm{NeD}$ & NMD & & MD & $\mathrm{NeD}$ & NMD \\
\hline$p_{1}$ & 9.34 & 6.22 & 3.33 & $p_{1}$ & 0.8 & 0.7 & 0.8 & $p_{1}$ & 0.36 & 0.43 & 0.21 \\
\hline$p_{2}$ & 9.38 & 6.30 & 3.64 & $p_{2}$ & 0.8 & 0.8 & 0.9 & $p_{2}$ & 0.36 & 0.29 & 0.36 \\
\hline$p_{3}$ & 8.67 & 6.03 & 3.14 & $p_{3}$ & 0.5 & 0.7 & 0.7 & $p_{3}$ & 0.21 & 0.43 & 0.36 \\
\hline$p_{4}$ & 9.73 & 6.66 & 3.90 & $p_{4}$ & 0.9 & 0.9 & 0.9 & $p_{4}$ & 0.57 & 0.36 & 0.07 \\
\hline$p_{5}$ & 9.50 & 6.50 & 3.03 & $p_{5}$ & 0.8 & 0.8 & 0.7 & $p_{5}$ & 0.21 & 0.36 & 0.43 \\
\hline \multicolumn{5}{|c|}{ AM of dyspnea degrees } & \multicolumn{3}{|c|}{$\mathrm{MD}, \mathrm{NeD}$, and NMD values } & \multicolumn{4}{|c|}{ Phase terms } \\
\hline
\end{tabular}

\begin{tabular}{llllllllll}
\hline & 0.1 & 0.2 & 0.3 & 0.4 & 0.5 & 0.6 & 0.7 & 0.8 & 0.9 \\
\hline MD & 7.3 & 7.6 & 7.9 & 8.2 & 8.5 & 8.8 & 9.1 & 9.4 & 9.7 \\
NeD & 4.3 & 4.6 & 4.9 & 5.2 & 5.5 & 5.8 & 6.1 & 6.4 & 6.7 \\
NMD & 1.3 & 1.6 & 1.9 & 2.2 & 2.5 & 2.8 & 3.1 & 3.4 & 3.7 \\
\hline
\end{tabular}

\begin{tabular}{|c|c|c|c|c|c|c|c|c|c|c|c|}
\hline & MD & $\mathrm{NeD}$ & NMD & & MD & $\mathrm{NeD}$ & NMD & & MD & $\mathrm{NeD}$ & NMD \\
\hline$p_{1}$ & 8.37 & 5.94 & 3.48 & $p_{1}$ & 0.5 & 0.6 & 0.8 & $p_{1}$ & 0.21 & 0.36 & 0.43 \\
\hline$p_{2}$ & 9.67 & 6.63 & 3.90 & $p_{2}$ & 0.8 & 0.9 & 0.9 & $p_{2}$ & 0.50 & 0.43 & 0.07 \\
\hline$p_{3}$ & 9.73 & 6.52 & 3.47 & $p_{3}$ & 0.9 & 0.8 & 0.8 & $p_{3}$ & 0.43 & 0.36 & 0.21 \\
\hline$p_{4}$ & 9.78 & 6.80 & 3.14 & $p_{4}$ & 0.9 & 0.9 & 0.7 & $p_{4}$ & 0.36 & 0.29 & 0.36 \\
\hline$p_{5}$ & 8.90 & 6.52 & 3.85 & $p_{5}$ & 0.6 & 0.8 & 0.9 & $p_{5}$ & 0.50 & 0.36 & 0.14 \\
\hline \multicolumn{4}{|c|}{ AM of cough degree } & & \multicolumn{3}{|c|}{$\mathrm{MD}, \mathrm{NeD}$, and NMD values } & \multicolumn{4}{|c|}{ Phase terms } \\
\hline
\end{tabular}

Table 17 CTSFSs obtained using CTSDFWAA and CTSDFWGA

\begin{tabular}{llr}
\hline & $C T S D F W A A$ & $C T S D F W G A$ \\
\hline$p_{1}$ & $\left(0.709 e^{i 2 \pi 0.429}, 0.448 e^{i 2 \pi 0.368}, 0.655 e^{i 2 \pi 0.174}\right)$ & $\left(0.520 e^{i 2 \pi 0.256}, 0.448 e^{i 2 \pi 0.368}, 0.769 e^{i 2 \pi 0.359}\right)$ \\
$p_{2}$ & $\left(0.752 e^{i 2 \pi 0.524}, 0.559 e^{i 2 \pi 0.319}, 0.851 e^{i 2 \pi 0.076}\right)$ & $\left(0.790 e^{i 2 \pi 0.405}, 0.559 e^{i 2 \pi 0.319}, 0.880 e^{i 2 \pi 0.312}\right)$ \\
$p_{3}$ & $\left(0.788 e^{i 2 \pi 0.364}, 0.449 e^{i 2 \pi 0.368}, 0.538 e^{i 2 \pi 0.254}\right)$ & $\left(0.112 e^{i 2 \pi 0.080}, 0.449 e^{i 2 \pi 0.368}, 0.704 e^{i 2 \pi 0.509}\right)$ \\
$p_{4}$ & $\left(0.875 e^{i 2 \pi 0.424}, 0.449 e^{i 2 \pi 0.239}, 0.849 e^{i 2 \pi 0.299}\right)$ & $\left(0.875 e^{i 2 \pi 0.424}, 0.449 e^{i 2 \pi 0.239}, 0.849 e^{i 2 \pi 0.299}\right)$ \\
$p_{5}$ & $\left(0.711 e^{i 2 \pi 0.418}, 0.449 e^{i 2 \pi 0.315}, 0.337 e^{i 2 \pi 0.171}\right)$ & $\left(0.245 e^{i 2 \pi 0.080}, 0.449 e^{i 2 \pi 0.315}, 0.831 e^{i 2 \pi 0.574}\right)$ \\
\hline
\end{tabular}


Table 18 Score values for CTSDFWAA values

\begin{tabular}{lllllllllll}
\hline & $\eta=1$ & $\eta=2$ & $\eta=3$ & $\eta=4$ & $\eta=5$ & $\eta=6$ & $\eta=7$ & $\eta=8$ & $\eta=9$ & $\eta=10$ \\
\hline$P_{1}$ & 0.505 & 0.515 & 0.510 & 0.523 & 0.524 & 0.525 & 0.526 & 0.527 & 0.527 & 0.528 \\
$P_{2}$ & 0.460 & 0.475 & 0.483 & 0.488 & 0.490 & 0.492 & 0.493 & 0.494 & 0.495 & 0.496 \\
$P_{3}$ & 0.528 & 0.554 & 0.567 & 0.574 & 0.578 & 0.581 & 0.583 & 0.585 & 0.586 & 0.587 \\
$P_{4}$ & $\mathbf{0 . 5 5 9}$ & $\mathbf{0 . 5 7 6}$ & $\mathbf{0 . 5 8 3}$ & $\mathbf{0 . 5 8 7}$ & $\mathbf{0 . 5 8 9}$ & $\mathbf{0 . 5 9 1}$ & $\mathbf{0 . 5 9 2}$ & $\mathbf{0 . 5 9 3}$ & $\mathbf{0 . 5 9 4}$ & $\mathbf{0 . 5 9 4}$ \\
$P_{5}$ & 0.511 & 0.520 & 0.524 & 0.526 & 0.527 & 0.528 & 0.529 & 0.530 & 0.530 & 0.531 \\
\hline
\end{tabular}

Table 19 Score values for CTSDFWGA values

\begin{tabular}{lllllllllll}
\hline & $\eta=1$ & $\eta=2$ & $\eta=3$ & $\eta=4$ & $\eta=5$ & $\eta=6$ & $\eta=7$ & $\eta=8$ & $\eta=9$ & $\eta=10$ \\
\hline$P_{1}$ & 0.477 & 0.473 & 0.471 & 0.470 & 0.469 & 0.469 & 0.469 & 0.468 & 0.468 & 0.468 \\
$P_{2}$ & 0.449 & 0.436 & 0.429 & 0.425 & 0.422 & 0.420 & 0.419 & 0.418 & 0.418 & 0.417 \\
$P_{3}$ & 0.489 & 0.482 & $\mathbf{0 . 4 7 8}$ & $\mathbf{0 . 4 7 5}$ & $\mathbf{0 . 4 7 3}$ & $\mathbf{0 . 4 7 2}$ & $\mathbf{0 . 4 7 1}$ & $\mathbf{0 . 4 7 0}$ & $\mathbf{0 . 4 7 0}$ & $\mathbf{0 . 4 6 9}$ \\
$P_{4}$ & $\mathbf{0 . 5 1 8}$ & $\mathbf{0 . 4 9 0}$ & 0.474 & 0.464 & 0.459 & 0.455 & 0.452 & 0.450 & 0.448 & 0.447 \\
$P_{5}$ & 0.451 & 0.429 & 0.420 & 0.414 & 0.411 & 0.409 & 0.408 & 0.407 & 0.406 & 0.405 \\
\hline
\end{tabular}

Step 3: Using Eq. 1, score values of the aggregated values are obtained as follows:

\begin{tabular}{lll}
\hline & $\begin{array}{l}\text { SVs according to } \\
\text { CTSDFWAA op. }\end{array}$ & $\begin{array}{l}\text { SVs according to } \\
\text { CTSDFWGA op. }\end{array}$ \\
\hline$p_{1}$ & 0.505 & 0.447 \\
$p_{2}$ & 0.460 & 0.449 \\
$p_{3}$ & 0.528 & 0.489 \\
$p_{4}$ & $\mathbf{0 . 5 5 9}$ & $\mathbf{0 . 5 1 8}$ \\
$p_{5}$ & 0.511 & 0.451 \\
\hline
\end{tabular}

Step 4: According to Table 4.1, $p_{4}$ is suffer from COVID-19.

\section{Sensitivity analyses and discussion}

In this section, we compute the score values of the patients according to CTSDFWAA and CTSDFWGA values for " $\eta=$ $1,2, \ldots, 10 "$ ".

According to Tables 18 and 19, we see that for $\eta=$ $1,2, \ldots, 10$, results obtained using CTSDFWAA operator match by medical results. By results, $P_{4}$ was infected by COVID-19. For $\eta=1,2$ results from both operators are consistent. For $\eta=3,4, \ldots, 10$ according to results obtained using CTSDFWGA operator $P_{3}$ was infected by COVID- 19 . Also, this matches the medical results made by a medical doctor. In this study, we consider only four symptoms. However, the epidemic of the COVID-19 continue all over the worlds and medical researchers encounter some new symptoms of COVID-19. Here we give a simple example to show the trueness of the proposed method, this is a restriction of our study. We think that this study may be a reference point for researchers who want to study clustering and medical diagnosis with large data.

\section{Conclusion}

In this paper, weakness of score and accuracy function defined by Ali et al. [57] was pointed out and new score and accuracy functions were defined for CTSFNs. Set theoretical operations was introduced and some aggregation operators based on Dombi t-norms and t-conorms were defined under CTSF environment with their examples. Also, some properties of the proposed aggregation operators were investigated. Furthermore, an MCDM method was developed based on proposed aggregation operators and score function. Moreover, an application of the developed method, including determining the COVID-19, was presented by transforming the real data to CTSF data. We see that obtained results match real results. We also pointed out some restrictions and their reasons. In future, our targets are to study other aggregation operators such as Hamacher and Bonferroni, similarity measures, distance measures and decision-making methods based on TOPSIS, VIKOR, AHP, etc. By transferring the algorithm of the proposed method to the computer program, our analysis for a limited number of patients can be made under big data and by considering more parameters. We hope that this study will provide a useful perspective for researchers working on decision-making.

Acknowledgements We thank Dr. Ahmed Qasim Al-Rawi who the manager of Heet hospital in Al-Anbar, Iraq, and Dr. Omer Moayed AlBayati who is a doctor in Al Mahmoodia hospital in Baghdad for real data and information. In this study, we got patients' permissions and attached the signed documents that allow the use of patient data. 


\section{Declarations}

Conflict of interest The authors declare no conflict of interest.

Open Access This article is licensed under a Creative Commons Attribution 4.0 International License, which permits use, sharing, adaptation, distribution and reproduction in any medium or format, as long as you give appropriate credit to the original author(s) and the source, provide a link to the Creative Commons licence, and indicate if changes were made. The images or other third party material in this article are included in the article's Creative Commons licence, unless indicated otherwise in a credit line to the material. If material is not included in the article's Creative Commons licence and your intended use is not permitted by statutory regulation or exceeds the permitted use, you will need to obtain permission directly from the copyright holder. To view a copy of this licence, visit http://creativecomm ons.org/licenses/by/4.0/.

\section{References}

1. Zadeh LA (1965) Fuzzy sets. Inf Control 8(3):338-353

2. Atanassov KT (1986) Intuitionistic fuzzy sets. Fuzzy Sets Syst 20(1):87-96

3. Yager RR (2013) Pythagorean fuzzy subsets. IEEE 2013:57-61

4. Yager RR (2013) Pythagorean membership grades in multi-criteria decision making. IEEE Trans Fuzzy Syst 22(4):958-965

5. Yager RR (2017) Generalized orthopair fuzzy sets. IEEE Trans Fuzzy Syst 25:1222-1230

6. Cuong BC (2013) Picture fuzzy sets-First results, Part 1. In: Seminar Neuro-Fuzzy Systems with Applications; Institute of Mathematics, Vietnam Academy of Science and Technology: Hanoi, Vietnam (2013)

7. Cuong BC (2013) Picture fuzzy sets-First results, Part 2. In: Seminar Neuro-Fuzzy Systems with Applications; Institute of Mathematics, Vietnam Academy of Science and Technology: Hanoi, Vietnam (2013)

8. Garg H (2017) Some picture fuzzy aggregation operators and their applications to multicriteria decision-making. Arab J Sci Eng 42(12):5275-5290

9. Peng X, Dai J (2017) Algorithm for picture fuzzy multiple attribute decision-making based on new distance measure. Int J Uncertain Quantif 7(2): 177-187

10. Wei G (2017) Picture fuzzy aggregation operators and their application to multiple attribute decision making. J Intell Fuzzy Syst 33(2):713-724

11. Wei G (2018) TODIM method for picture fuzzy multiple attribute decision making. Informatica 29:555-566

12. Cao G (2020) A multi-criteria picture fuzzy decision-making model for green supplier selection based on fractional programming. Int J Comput Commun Control 15(1):1-14

13. Joshi R (2020) A novel decision-making method using r-norm concept and VIKOR approach under picture fuzzy environment. Expert Syst Appl 147:113228

14. Tian C, Peng J, Zhang W, Zhang S, Wang J (2020) Tourism environmental impact assessment based on improved AHP and picture fuzzy PROMETHEE II methods. Technol Econ Dev Econ 26(2):355-378

15. Wei G (2017) Some cosine similarity measures for picture fuzzy sets and their applications to strategic decision making. Informatica 28(3):547-564
16. Wei G, Gao H (2018) The generalized Dice similarity measures for picture fuzzy sets and their applications. Informatica 29(1):107124

17. Wei G (2018) Some similarity measures for picture fuzzy sets and their applications. Iran J Fuzzy Syst 15(1):77-89

18. Rafiq M, Ashraf S, Abdullah S, Mahmood T, Muhammad S (2019) The cosine similarity measures of spherical fuzzy sets and their applications in decision making. J Intell Fuzzy Syst 36(6):60596073

19. Thao NX (2020) Similarity measures of picture fuzzy sets based on entropy and their application in MCDM. Pattern Anal Appl 23(3):1203-1213

20. Singh P (2015) Correlation coefficients for picture fuzzy sets. J Intell Fuzzy Syst 28(2):591-604

21. Ganie AH, Singh S, Bhatia PK (2020) Some new correlation coefficients of picture fuzzy sets with applications. Neural Comput Appl 32:12609-12625

22. Son LH (2016) Generalized picture distance measure and applications to picture fuzzy clustering. Appl Soft Comput 46(C):284-295

23. Hao ND, Son LH, Thong PH (2016) Some improvements of fuzzy clustering algorithms using picture fuzzy sets and applications for geographic data clustering. VNU J Sci Comput Sci Commun Eng 32(3):32-38

24. Gündogdu FK, Kahraman C (2019) Spherical fuzzy sets and spherical fuzzy TOPSIS method. J Intell Fuzzy Syst 36(1):337-352

25. Gündogdu FK, Kahraman C (2019) Spherical fuzzy sets and decision making applications. In: Kahraman C, Cebi S, Cevik Onar S, Oztaysi B, Tolga A, Sari I (eds) Intelligent and fuzzy techniques in big data analytics and decision making. INFUS 2019. Advances in Intelligent Systems and Computing, p 1029. Springer, Cham

26. Mahmood T, Ullah K, Khan Q, Jan N (2019) An approach toward decision-making and medical diagnosis problems using the concept of spherical fuzzy sets. Neural Comput Appl 31:7041-7053

27. Ullah K, Mahmood T, Jan N (2018) Similarity Measures for TSpherical Fuzzy Sets with Applications in Pattern Recognition. Symmetry 10(6): 193

28. Garg H, Munir M, Ullah K, Mahmood T, Jan N (2018) Algorithm for T-spherical fuzzy multi-attribute decision making based on improved interactive aggregation operators. Symmetry 10(12):670

29. Ullah K, Mahmood T, Jan N, Ali Z (2018) A note on geometric aggregation operators in T-spherical fuzzy environment and their applications in multi-attribute decision making. J Eng Appl Sci 37(2):75-86

30. Wu M, Chen T, Fan J (2020) Divergence measure of T-spherical fuzzy sets and its applications in pattern recognition. IEEE Access $8: 10208-10221$

31. Ullah K, Hassan N, Mahmood T, Jan N, Hassan M (2019) Evaluation of investment policy based on multi-attribute decision-making using interval valued T-spherical fuzzy aggregation operators. Symmetry 11(3):357

32. Liu P, Khan Q, Mahmood T, Hassan N (2019) T-spherical fuzzy power muirhead mean operator based on novel operational laws and their application in multi-attribute group decision making. IEEE Access 7:22613-22632

33. Guleria A, Bajaj RK (2019) T-spherical fuzzy soft sets and its aggregation operators with application in decision making. Sci Iran. https://doi.org/10.24200/SCI.2019.53027.3018

34. Quek SG, Selvachandran G, Munir M, Mahmood T, Ullah K, Son LH, Thong PH, Kumar R, Priyadarshini I (2019) Multi-attribute multi-perception decision-making based on generalized t-spherical fuzzy weighted aggregation operators on neutrosophic sets. Mathematics 7:780

35. Munir M, Kalsoom H, Ullah K, Mahmood T, Chu YM (2020) T-spherical fuzzy Einstein hybrid aggregation operators and their applications in multi-attribute decision making problems. Symmetry $12(3): 365$ 
36. Ullah K, Garg H, Mahmood T, Jan N, Ali Z (2020) Correlation coefficients for T-spherical fuzzy sets and their applications in clustering and multi-attribute decision making. Soft Comput 24(3): 1647-1659

37. Ullah K, Mahmood T, Garg H (2020) Evaluation of the performance of search and rescue robots using t-spherical fuzzy hamacher aggregation operators. Int J Fuzzy Syst 22(2):570-582

38. Garg H, Ullah K, Mahmood T, Hassan N, Jan N (2021) T-spherical fuzzy power aggregation operators and their applications in multiattribute decision making. J Ambient Intell Hum Comput. https:// doi.org/10.1007/s12652-020-02600-z

39. Ju Y, Liang Y, Lu C, Dong P, Gonzalez EDS, Wang A (2021) Tspherical fuzzy TODIM method for multi-criteria group decisionmaking problem with incomplete weight information. Soft Comput 25(4):2981-3001

40. Chen Y, Munir M, Mahmood T, Hussain A, Zeng S (2021)some generalized t-spherical and group-generalized fuzzy geometric aggregation operators with application in MADM problems. J Math 2021

41. Munir M, Mahmood T (2021) Hussain A (2021) Algorithm for T-spherical fuzzy MADM based on associated immediate probability interactive geometric aggregation operators. Artif Intell Rev. https://doi.org/10.1007/s10462-021-09959-1

42. Ramot D, Milo R, Fiedman M, Kandel A (2002) Complex fuzzy sets. IEEE Trans Fuzzy Syst 10(2):171-186

43. Ramot D, Friedman M, Langholz G, Kandel A (2003) Complex fuzzy logic. IEEE Trans Fuzzy Syst 11(4):450-461

44. Alkouri A, Salleh A (2012) Complex intuitionistic fuzzy sets. In: International conference on fundamental and applied sciences, AIP conference proceedings, vol 1482, pp 464-470

45. Rani D, Garg H (2017) Distance measures between the complex intuitionistic fuzzy sets and their applications to the decisionmaking process. Int J Uncertainty Quant 7:5

46. Rani D, Garg H (2018) Complex intuitionistic fuzzy power aggregation operators and their applications in multicriteria decisionmaking. Expert Syst 35(6):e12325

47. Garg H, Rani D (2019) Some generalized complex intuitionistic fuzzy aggregation operators and their application to multicriteria decision-making process. Arab J Sci Eng 44(3):2679-2698

48. Garg H, Rani D (2019) Novel aggregation operators and ranking method for complex intuitionistic fuzzy sets and their applications to decision-making process. Artif Intell Rev 2019:1-26

49. Garg H, Rani D (2019) Exponential, logarithmic and compensative generalized aggregation operators under complex intuitionistic fuzzy environment. Group Decis Negot 28(5):991-1050

50. Garg H, Rani D (2019) Multi-criteria decision making method based on Bonferroni mean aggregation operators of complex intuitionistic fuzzy numbers. J Ind Manag Optim. https://doi.org/10. 3934/jimo.2020069

51. Garg H, Rani D (2020) Generalized geometric aggregation operators based on t-norm operations for complex intuitionistic fuzzy sets and their application to decision-making. Cogn Comput 12:679-698

52. Ullah K, Mahmood T, Ali Z, Jan N (2019) On some distance measures of complex Pythagorean fuzzy sets and their applications in pattern recognition. Complex Intell Syst 6:15-27

53. Liu P, Mahmood T, Ali Z (2020) Complex q-Rung orthopair fuzzy aggregation operators and their applications in multi-attribute group decision making. Information 11(5):2-28. https://doi.org/ 10.3390/info11010005

54. Akram M, Bashir A, Garg H (2020) Decision-making model under complex picture fuzzy Hamacher aggregation operators. Comput Appl Math 39(3):1-38
55. Liu P, Akram M, Bashir A (2021) Extensions of power aggregation operators for decision making based on complex picture fuzzy knowledge. J Intell Fuzzy Syst 40(1):1107-1128

56. Akram M, Garg H, Zahid K (2020) Extensions of ELECTRE-I and TOPSIS methods for group decision-making under complex Pythagorean fuzzy environment. Iran J Fuzzy Syst 17(5):147-164

57. Ali Z, Mahmood T, Yang MS (2020) Complex T-spherical fuzzy aggregation operators with application to multi-attribute decision making. Symmetry 12(8):1311

58. Akram M, Kahraman C, Zahid K (2021) Group decision-making based on complex spherical fuzzy VIKOR approach. Knowl-Based Syst 216:106793

59. Nasir A, Jan N, Yang MS, Khan SU (2021) Complex T-spherical fuzzy relations with their applications in economic relationships and international trades. IEEE Access. https://doi.org/10.1109/ ACCESS.2021.3074557

60. Dombi J (1982) A general class of fuzzy operators, the De Morgan class of fuzzy operators and fuzziness measures induced by fuzzy operators. Fuzzy Sets Syst 8(2):149-163

61. Liu P, Liu J, Chen SM (2018) Some intuitionistic fuzzy Dombi Bonferroni mean operators and their application to multi-attribute group decision making. J Oper Res Soc 69(1):1-24

62. Akram M, Dudek WA, Dar JM (2019) Pythagorean Dombi fuzzy aggregation operators with application in multicriteria decisionmaking. Int J Intell Syst 34(11):3000-3019

63. Waseem N, Akram M, Alcantud JCR (2019) Multiattribute decision-making based on m-polar fuzzy Hamacher aggregation operators. Symmetry 11(12):1498

64. Shahzadi G, Akram M, Al-Kenani AN (2020) Decision making approach under Pythagorean fuzzy Yager weighted operators. Mathematics 8(1):70

65. Jana C, Pal M, Wang JQ (2019) Bipolar fuzzy Dombi aggregation opeartors and its application in multiple-attribute decision-making process. J Ambient Intell Hum Comput 10:3533-3549

66. Jana C, Senapati T, Pal M, Yager RR (2019) Picture fuzzy Dombi aggregation operators; application to MADM process. Appl Soft Comput 74:99-109

67. Ashraf S, Abdullah S, Mahmood T (2020) Spherical fuzzy Dombi aggregation operators and their application in group decision making problems. J Ambient Intell Hum Comput 11:2731-2749

68. Akram M, Khan A (2020) Complex Pythagorean Dombi fuzzy graphs for decision making. Granul Comput. https://doi.org/10. 1007/s41066-020-00223-5

69. Akram M, Khan A, Karaaslan F (2021) Complex spherical Dombi fuzzy aggregation operators for decision-making. J Multiplevalued Logic Soft Comput (Accepted)

70. Wu Y-c, Chen C-s, Chan Y-j (2020) The outbreak of COVID-19: an overview. J Chin Med Assoc 83(3):217-220

71. Rafiq M, Macías-Díaz JE, Raza A, Ahmed N (2021) Design of a nonlinear model for the propagation of COVID-19 and its efficient nonstandard computational implementation. Appl Math Model 89:1835-1846

72. Center for systems science and engineering at Johns Hopkins university, COVID-19 dashboard, (2020) https:// gisanddata.maps.arcgis.com/apps/opsdashboard/index.html\#/ bda7594740fd40299423467b48e9ecf6

73. Shatanawi W, Raza A, Arif MS, Abodayeh K, Rafiq M, Bibi M (2020) Design of nonstandard computational method for stochastic susceptible-infected-treated-recovered dynamics of coronavirus model. Adv Differ Equ 1:505. https://doi.org/10.1186/s13662020-02960-y 
74. Macías-Díaz JE, Raza A, Ahmed N (2021) Rafiq M (2021) Analysis of a nonstandard computer method to simulate a nonlinear stochastic epidemiological model of coronavirus-like diseases. Comput Methods Programs Biomed. https://doi.org/10.1016/j.cmpb.2021. 106054

75. Akgül A, Ahmed N, Ali Raza, Iqbal Z, Rafiq M, Baleanu D, Azizur Rehman M (2021) New applications related to Covid-19. Result Phys 20:103663
Publisher's Note Springer Nature remains neutral with regard to jurisdictional claims in published maps and institutional affiliations. 